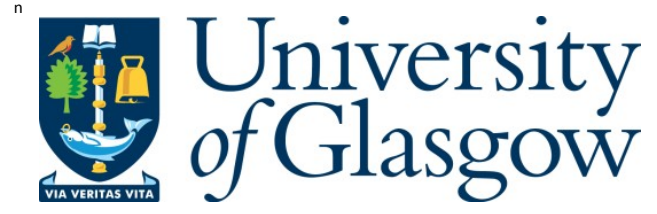

Colonia, S., Steijl, R., and B arakos, G. (2016) K inetic models and gas-kinetic schemes for hybrid simulation of partially rarefied flows. A IA A J ournal, (doi:10.2514/1.J 054137).

There may be differences between this version and the published version. $Y$ ou are advised to consult the publisher's version if you wish to cite from it.

http://eprints.gla.ac.uk/116450/

Deposited on: 03 M arch 2016

Enlighten - R esearch publications by members of the U niversity of Glasgow http://eprints.gla.ac.uk 


\title{
Kinetic Models and Gas Kinetic Schemes for Hybrid Simulation of Partially Rarefied Flows
}

\author{
S. Colonia, R. Steijl and G.Barakos \\ Aerospace Sciences Division \\ School of Engineering, University of Glasgow
}

\begin{abstract}
Approaches to predict flow fields that display rarefaction effects incur a cost in computational time and memory considerably higher than methods commonly employed for continuum flows. For this reason, to simulate flow fields where continuum and rarefied regimes coexist, hybrid techniques have been introduced. In the present work analytically defined Gas-Kinetic schemes based on the Shakhov and Rykov models, for monoatomic and diatomic gas flows, respectively, are proposed and evaluated with the aim to be used in the context of hybrid simulations. This should reduce the region where more expensive methods are needed by extending the validity of the continuum formulation. Moreover, since for rarefied gas flows at high velocities it is necessary to take into account the non-equilibrium among the internal degrees of freedom, the extension of the approach to employ diatomic gas models with rotational relaxation is a mandatory first step towards realistic simulations. Compared to previous works of $\mathrm{Xu}$ and co-workers the presented scheme is defined directly on the basis of kinetic models which involve a Prandtl number correction. Moreover, the methods are defined fully analytically instead of making use of Taylor expansion for the evaluation of the required derivatives. The scheme has been tested for various test cases and Mach numbers proving to produce reliable predictions in agreement with other approaches for near-continuum flows. Finally, the performance of the scheme, in terms of memory and computational time, compared to discrete velocity methods makes it a compelling alternative in place of more complex methods for hybrid simulations of weakly rarefied flows.
\end{abstract}




\section{Acronyms}

$\begin{array}{llll}\text { MФC } & \text { Multi-Physics Code } & \text { CE } & \text { Chapman-Enskog } \\ \text { BTE } & \text { Boltzmann Transport Equation } & \text { ES } & \text { Ellipsoidal-Statistical } \\ \text { NS } & \text { Navier-Stokes equations } & \text { DVM } & \text { Discrete Velocity Method } \\ \text { MD } & \text { Molecular Dynamics } & \text { DSMC } & \text { Direct Simulation Monte Carlo } \\ \text { UGKS } & \text { Unified Gas Kinetic Scheme } & \text { S-UGKS } & \text { UGKS based on the Shakhov model } \\ \text { BGK-NS } & \text { Gas-Kinetic BGK scheme for the NS equations } & \text { GKS } & \text { Gas Kinetic Scheme } \\ \text { R-GKS } & \text { GKS based on the Rykov model } & \text { S-GKS } & \text { GKS based on the Shakhov model } \\ \text { GKS } \tau^{*} & \text { GKS with modified } \tau & \text { AUSM } & \text { Advection Upstream Splitting Method }\end{array}$

\section{Nomenclature}

$\begin{array}{ll}t & \text { Time variable } \\ \rho & \text { Density } \\ T & \text { Total temperature } \\ T_{r} & \text { Rotational temperature } \\ p_{t} & \rho T_{t} \text { (non-dimensional translational pressure) } \\ q_{x} & \text { Total heat flux in i-direction } \\ q_{x}^{r} & \text { Rotational heat flux in x-direction } \\ \lambda & \text { Particle mean free path } \\ \mu & \text { Viscosity } \\ c_{x} & \text { Particle velocity in x-direction } \\ \zeta & \text { Rotational degrees of freedom } \\ \triangle x & \text { Spatial step } \\ f & \text { Particle distribution function } \\ \hat{\phi} & \text { Non-dimensional generic macroscopic variable } \\ \mathbf{W} & \text { Conservative variables vector } \\ \boldsymbol{\Psi} & \left(1, c_{x}, \mathbf{c}^{2}\right)^{T} \\ \boldsymbol{\Psi}_{0} & \left(1, c_{x}, \mathbf{c}^{2}, 0\right)^{T} \\ c_{p} & \text { Pressure coefficient }\end{array}$

$\mathbf{x} \quad$ Spatial coordinates vector

$u_{x} \quad$ Velocity in $\mathrm{x}$-direction

$T_{t} \quad$ Translational temperature

$p \quad \rho T$ (non-dimensional equilibrium pressure)

$p_{r} \quad \rho T_{r}$ (non-dimensional rotational pressure)

$q_{x}^{t} \quad$ Translational heat flux in x-direction

$m \quad$ Molecular mass

$\tau \quad$ Particle collision time

$Z_{r} \quad$ Collision number

$c_{x}^{\prime} \quad c_{i}-u_{i}$, particle velocity fluctuation

$\Delta t \quad$ Time step

$\Delta c_{x} \quad$ Velocity step

$F \quad m f$

$\phi \quad$ Generic macroscopic variable

$\mathbf{W}_{S / R} \quad$ Conservative variables, Shakhov/Rykov model

$\boldsymbol{\Psi}^{\prime} \quad\left(1, c_{x}, \mathbf{c}^{2}, \zeta^{2}\right)^{T}$

$\Psi_{1} \quad(0,0,1,1)^{T}$

$R \quad$ Gas constant 


\section{Introduction}

At intermediate altitudes $(70-90 \mathrm{Km})$, the flow around hypersonic aircraft can be characterised as mainly continuum with localised areas (generated by the rapid expansion in the wake of the vehicle as well as by strong gradients in shock waves and boundary layers) that display rarefaction effects. The flow conditions near the vehicle surface and in the wake determine the drag and the heat transferred to the vehicle and its payload. It is therefore important that these regions are simulated using appropriate physical models. When the gradients of the macroscopic variables become so steep that their length scale is of the same order as the average distance travelled by molecules between collisions, the number of impacts is not enough to drive the fluid towards a local thermodynamic equilibrium. At these conditions the flow can no longer be considered a continuum and the transport terms in the Navier-Stokes equations (NS) fail since the constitutive relation is not valid.

The mathematical model at molecular level is the Boltzmann equation (BTE) ${ }^{43}$, and for the regions of the flow field where highly non-equilibrium effects occur the Direct Simulation Monte Carlo method (DSMC) ${ }^{6}$ is typically employed to statistically estimate the solution of the BTE. Alternatively a discrete velocity method (DVM) ${ }^{3,40}$ can be used to solve a kinetic model approximation of the BTE ${ }^{4,31,35}$.

In previous works ${ }^{7,21,25,44}$ hybrid techniques have been introduced to simulate flow fields where continuum and rarefied regimes coexist. In these methods, the more expensive approach, DSMC or DVM, is employed only where needed and is coupled with a finite-volume scheme for the NS equations used where the flow is continuum. A hybrid technique couples two different simulation methods by means of information exchange between the parts of the flow domain. In recent works, this has been achieved using an overlap region where flow state variables or numerical fluxes are exchanged between the two models ${ }^{34}$ or employing a buffer region where the two models are blended at equation level ${ }^{18}$. Recent works on these methods focused on rarefied high-Mach flow can be found in ${ }^{16,17,19}$.

An alternative approach is presented in ${ }^{26,50}$. Indeed, the Unified Gas-Kinetic Scheme (UGKS) ${ }^{26,50}$ uses a finite-volume method where the numerical fluxes are based on the solution of the Shakhov model ${ }^{35}$ for a monoatomic gas, or the Rykov model ${ }^{31}$ for a diatomic gas with rotational non-equilibrium. Where the flow is under-resolved, by accounting for the pressure jump in the definition of the collision time, additional numerical viscosity is added resulting in a shock thickness of the order of the cell size ${ }^{45}$. This allows the UGKS to simulate flows in both rarefied and continuum regimes.

In the present work, two GKS methods, analytically defined on the basis of the Chapman-Enskog (CE) expansion of non-dimensional Shakhov and Rykov models, are proposed to simulate weakly rarefied flows. The derivatives of the equilibrium function and the time derivatives of the primitive variables are defined analytically employing the compatibility condition of the kinetic model for the latter. In previous works from Xu and co-workers ${ }^{48,51}$ similar gas-kinetic schemes are defined using the CE solution of the BGK model with and without rotational non-equilibrium and a scaling of the energy numerical flux ${ }^{45}$ to correct the Prandtl number. Moreover, in those schemes the required derivative are expressed in terms of Taylor series where the coefficient are calculated by means of properties of the employed BGK model. The proposed GKS, due to the use of the CE expansion, is limited to near-continuum regions but is simpler than the UGKS ${ }^{26,50}$. However, the validity of the approach can be extended considering a modified collision time ${ }^{46}$. Also this correction, in the present work, is defined fully analytically for both schemes.

Based on the literature survey of related works, the authors believe that the proposed GKS represents an efficient method, relative to DVM, capable of modelling complex diatomic gas flows with moderate rarefaction effects but with significant rotational non-equilibrium. As such the proposed approach is a novel alternative to the DVM for a range of practically relevant flows. Moreover, the update of the non-equilibrium distribution function, as used in the UGKS, is neglected reducing the memory cost of the approach. Thus, the GKS method represents a viable option to reduce the cost of hybrid simulations by reducing the region where the expensive method is needed and extending the validity of the continuum formulation.

The schemes are built in a computational framework that also includes a DVM for the kinetic Boltzmann equations successfully employed for different monoatomic cases ${ }^{37}$. The framework has been recently improved ${ }^{14}$ with the addition of the Rykov model and an Ellipsoidal-Statistical (ES) model ${ }^{2}$ for diatomic gases with rotational nonequilibrium.

In part 2 the mathematical definition of the Rykov and Shakhov models and of the proposed GKS are described; while in part 3, an assessment of reliability and performance of the presented GKS for both monoatomic and diatomic flows is discussed. The discussion also includes a comparison with performance and results of the DVM implemented in the same framework in order to evaluate the use of the GKS in future works about hybrid simulation. 


\section{Flow Models and Numerical Schemes}

\subsection{Multi-Physics Code}

The methods used in the present work are built in the Multi-Physics Code (MФC) developed at the University of Liverpool $^{36-38}$. MФC is a computational framework designed for simulations of complex flows, where different mathematical flow models are employed for different regions of the flow domain depending on the flow physics. The NS equations represent the baseline level of mathematical models used. For the continuum flow solver based on the compressible NS equations, a cell-centred block-structured finite-volume method is employed using the AUSM ${ }^{+} /$up method for the convective fluxes ${ }^{13}$. For low-speed flow analysis, the framework further includes a Lattice Boltzmann Method as well as a Vortex-In-Cell method for vortex-dominated incompressible flows.

In the present work, emphasis is placed on the simulation of hypersonic, partially-rarefied flows for which mathematical models at a more detailed level of physics than the compressible NS are required. For flows with strong nonequilibrium and rarefied effects, the framework includes Molecular Dynamics (MD) methods as well as deterministic DVM for a range of kinetic Boltzmann equations. The Shakhov and ES models are included for monoatomic gas flows, while the Rykov model and a polyatomic ES-BGK model were implemented for diatomic gas flow simulations ${ }^{14}$. The

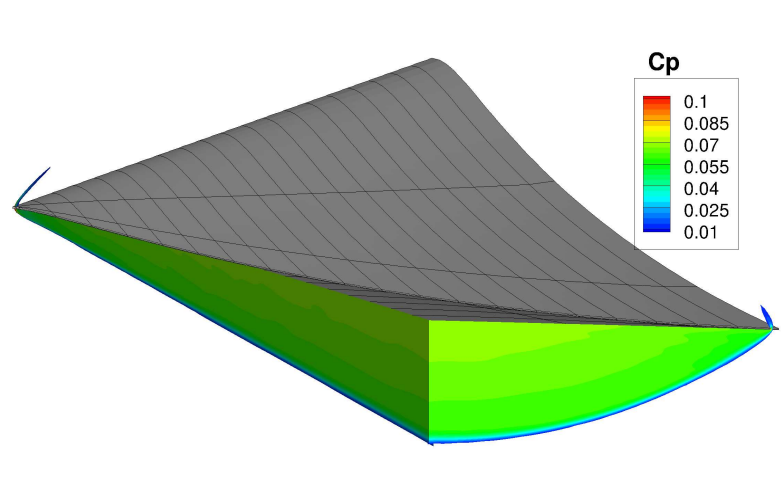

(a) Pressure distribution at Mach 8 .

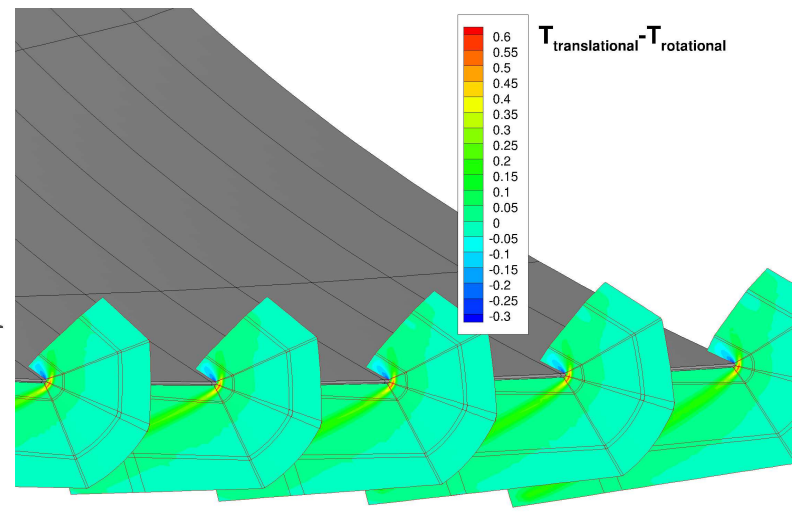

(b) Flow solution in kinetic-flow domain.

Figure 1 - Mach 8 Waverider test case; leading-edge diameter $5 \lambda\left(\operatorname{Knudsen}_{L E}=0.2\right)$ and body length $5 \times 10^{3} \lambda$.

kinetic models are discretised using a discrete velocity method within a finite-volume method for multi-block structured grids using-second order TVD time marching. The velocity (phase) space is discretised using either a uniformly spaced method using the trapezoidal rule for the evaluation of the moments of the distribution functions or a Gaussquadrature method with modified Hermite polynomials. For the present high-speed flow cases, the uniform velocity space with the trapezoidal rule is the preferred approach and was used exclusively.

The memory as well as CPU time requirements are considerable and therefore an efficient parallel implementation involving 'two' levels of parallelism was conceived. In this parallelisation strategy, the phase space as well as the flow domain are distributed over the processes. First, the phase space is partitioned in regular sub-spaces, each to be assigned to separate processes within separate MPI communicators. The overall number of processes is then divided by the number partitions to obtain the required number of communicators. The mesh-blocks in physical space are then equally distributed over these communicators.

For the coupling between the continuum solver and the kinetic solver, МФC employs state-based or flux-based hybrid techniques. While, for hybrid NS-DSMC simulations the choice of a state-based coupling is the preferable one due to the lower scattering error that it involves ${ }^{34}$, in the current deterministic DVM kinetic solver, the statistical scatter is absent, creating more flexibility in the used coupling technique.

The example shown in figures $1 \mathrm{a}$ and $1 \mathrm{~b}$ is for a Mach 8 flow of a diatomic gas around a waverider (leading-edge diameter $5 \lambda$ and body length $5 \times 10^{3} \lambda$ ) geometry investigated as part of a European Union funded project (PRACE Preparatory Access), providing access to the SuperMUC computer at the Leibniz Rechenzentrum (LRZ) in Munich. A multi-block mesh with 867 blocks and 3 million cells, of which 0.5 million kinetic, was used and 2048 cores of Intel Xeon processors, with $1 G B$ of memory each, were needed due to the memory usage. Computational and memory cost is a major drawback employing the DVM in hybrid simulation and the use of the GKS to reduce the extension of the domain where it is strictly required represents a preferable alternative. 


\subsection{Non-dimensional Shakhov Model}

The complex collision term in the right-hand side of the BTE ${ }^{11}$ poses a major challenge in modelling flows using this equation. For this reason, kinetic model equations have been developed which approximate the BTE such that the exact moment equations up to a desired order are respected. The first kinetic model equation to be proposed has been the Bhatnagar-Gross-Krook (BGK) model ${ }^{4}$ where the complex collision term has been replaced with a relaxation term towards the Maxwellian equilibrium (4).

It is well known ${ }^{43}$ that the BGK model recovers a unit Prandtl number in the continuum limit and for this reason various corrected models have been presented in the literature. Among them, the Shakhov model ${ }^{35}$ recovers the correct continuum limit Prandtl number by scaling the heat fluxes and has been proved to be a reliable kinetic approximation, up to the heat fluxes moments of the BTE, for flows where only translational non-equilibrium is considered ${ }^{12}$.

Defining the following non-dimensional variables

$$
\begin{array}{lll}
\rho=\hat{\rho} / \rho_{\infty} & ; \quad T=\hat{T} / T_{\infty} & ; \quad u=\hat{c} / \sqrt{2 R T_{\infty}} \\
t=\hat{t} /\left(\mu_{\infty} p_{\infty}^{-1}\right) & ; \quad \mathbf{x}=\hat{\mathbf{x}} /\left(\sqrt{2 R T_{\infty}} \mu_{\infty} p_{\infty}^{-1}\right) \quad ; \quad p=\hat{p} /\left(\rho_{\infty} R T_{\infty}\right) \\
\mu=\hat{\mu} / \mu_{\infty} \quad ; \quad q=\hat{q} /\left(\rho_{\infty}\left(2 R T_{\infty}\right)^{3 / 2}\right) \quad ; \quad \tau=\hat{\tau} /\left(\mu_{\infty} p_{\infty}^{-1}\right)
\end{array}
$$

where the dimensional variables are denoted with a hat and $c_{\infty}=\sqrt{2 R T_{\infty}}$ represents the most probable molecular velocity magnitude at equilibrium at the reference temperature $T_{\infty}$, the non-dimensional distribution function for the Shakhov model results

$$
F_{S}=\hat{F}_{S} /\left(\rho_{\infty}\left(2 R T_{\infty}\right)^{-3 / 2}\right) .
$$

Then, substituting the latter variables in the Shakhov model written in terms of $F=m f$ we obtain

$$
\begin{aligned}
& \frac{\partial F}{\partial t}+\mathbf{c} \frac{\partial F}{\partial \mathbf{x}}=\frac{F^{S}-F}{\tau} \\
& F_{S}=F_{M}(T)\left[1+\frac{8}{15} \frac{q_{x}}{p} \frac{c^{\prime}{ }_{x}}{T}\left(\frac{\mathbf{c}^{\prime 2}}{T}-\frac{5}{2}\right)\right]
\end{aligned}
$$

where

$$
F_{M}(T)=\frac{\rho}{(\pi T)^{3 / 2}} \exp \left(-\frac{\mathbf{c}^{\prime 2}}{T}\right)
$$

is the non-dimensional Maxwellian. The total collision time $\tau$ is expressed as $\mu / p$ where, in the present work, a power law with an exponential factor of $0.72^{22}$ is employed for the viscosity coefficient.

The dimensionless macroscopic quantities can be obtained from $F$ by means of the following moments

$$
\begin{array}{ll}
\rho=\int_{-\infty}^{+\infty} F d \mathbf{c} \quad ; \rho u_{x}=\int_{-\infty}^{+\infty} c_{x} F d \mathbf{c} ; \frac{3}{2} \rho T+\rho u_{x}^{2}=\int_{-\infty}^{+\infty} c^{2} F d \mathbf{c} \\
q_{x}=\int_{-\infty}^{+\infty} c^{\prime}{ }_{x} \frac{\mathbf{c}^{\prime 2}}{2} F d \mathbf{c} ; p=\rho T .
\end{array}
$$

\subsection{Non-dimensional Rykov Model}

Considering the flow of a diatomic gas, we will assume that the gas temperature is not too high, so that the vibrational degrees of freedom are not excited, and not too low, so that the rotational degrees of freedom are considered fully excited. In this case the particle distribution function $f(\mathbf{x}, \mathbf{c}, t, \zeta)$, which describes the state of the gas, will be a function not only of the spatial coordinate $\mathbf{x}$, the particle velocity $\mathbf{c}$ and the time $t$, but also of the rotational degrees of freedom $\zeta$. The Rykov model represents an extension of the Shakhov model where also rotational non-equilibrium is considered and has been proved to be a reliable kinetic approximation, up to the heat fluxes moments of the BTE, for this kind of flow $26,31-33$. Since the rotational degrees of freedom is considered fully excited, $\zeta$ is reduced by the model and a second distribution function is obtained. 
Employing the non-dimensional variables (1) the non-dimensional distribution functions of the model result

$$
F_{0}=\hat{F}_{0} /\left(\rho_{\infty}\left(2 R T_{\infty}\right)^{-3 / 2}\right) \quad ; \quad F_{1}=\hat{F}_{1} /\left(m R T_{\infty} \rho_{\infty}\left(2 R T_{\infty}\right)^{-3 / 2}\right)
$$

thus, for the Rykov model written in terms of $F=m f$ we obtain

$$
\begin{aligned}
& \frac{\partial F_{0}}{\partial t}+\mathbf{c} \frac{\partial F_{0}}{\partial \mathbf{x}}=\frac{F_{0}^{e q}-F_{0}}{\tau} \quad ; \quad \frac{\partial F_{1}}{\partial t}+\mathbf{c} \frac{\partial F_{1}}{\partial \mathbf{x}}=\frac{F_{1}^{e q}-F_{1}}{\tau} \\
& F_{0}^{e q}=\frac{1}{Z_{r}} F_{0}^{r}+\left(1-\frac{1}{Z_{r}}\right) F_{0}^{t} \quad ; \quad F_{1}^{e q}=\frac{1}{Z_{r}} F_{1}^{r}+\left(1-\frac{1}{Z_{r}}\right) F_{1}^{t} \\
& F_{0}^{r}=F_{M}(T)\left[1+\frac{8}{15} \omega_{0} \frac{q_{x}^{t}}{p} \frac{c^{\prime}{ }_{x}}{T}\left(\frac{\mathbf{c}^{\prime 2}}{T}-\frac{5}{2}\right)\right] \\
& F_{0}^{t}=F_{M}\left(T_{t}\right)\left[1+\frac{8}{15} \frac{q_{x}^{t}}{p_{t}} \frac{c^{\prime}{ }_{x}}{T_{t}}\left(\frac{\mathbf{c}^{\prime 2}}{T_{t}}-\frac{5}{2}\right)\right] \\
& F_{1}^{r}=T F_{M}(T)\left[1+\frac{8}{15} \omega_{0} \frac{q_{x}^{t}}{p} \frac{c_{x}^{\prime}{ }_{x}}{T}\left(\frac{\mathbf{c}^{\prime 2}}{T}-\frac{5}{2}\right)+4 \omega_{1}(1-\delta) \frac{q_{i}^{r} c^{\prime}{ }_{i}}{p T}\right] \\
& F_{1}^{t}=T_{r} F_{M}\left(T_{t}\right)\left[1+\frac{8}{15} \frac{q_{x}^{t}}{p_{t}} \frac{c^{\prime}{ }_{x}}{T_{t}}\left(\frac{\mathbf{c}^{\prime 2}}{T_{t}}-\frac{5}{2}\right)+4(1-\delta) \frac{q_{i}^{r} c^{\prime}{ }_{i}}{p_{t} T_{r}}\right]
\end{aligned}
$$

where

$$
F_{M}(T)=\frac{\rho}{(\pi T)^{3 / 2}} \exp \left(-\frac{\mathbf{c}^{\prime 2}}{T}\right)
$$

and the total collision time $\tau$ is expressed as $\mu_{t} / p_{t}$ with the viscosity determined from the translational temperature.

In a system of colliding particles, energy is transferred between the various internal modes. These collisions tend to drive the internal energy distributions towards their respective equilibrium state and the number of them necessary to push a particular mode to the equilibrium is the collision number, $Z$, associated to that mode ${ }^{8}$. The Rykov model is based on the assumption that the fraction of collisions involving the excitation of the rotational degrees of freedom, $Z_{r}$, is a given constant or a function of the flow temperatures. Several works provide an expression of $Z_{r}$ as a function of the temperature in the flow field. Probably the first attempt to appear in the literature is the theoretical work of Parker ${ }^{29}$ where, employing an empirical non impulsive model and assuming coplanar collisions and zero initial rotational energy, the following approximate expression is obtained

$$
Z_{r}^{\text {Par. }}=\frac{\left(Z_{r}\right)_{\infty}}{1+\left(\pi^{3 / 2} / 2\right)\left(T^{*} / T\right)^{1 / 2}+\left(\pi^{2} / 4+\pi\right)\left(T^{*} / T\right)}
$$

$T *=91.5 \mathrm{~K}$ is the characteristic temperature of the intermolecular potential and $\left(Z_{r}\right)_{\infty}=23.5$ is the limiting value suggested in ${ }^{5}$. While Parker's expression, (9), is derived involving a large number of simplifying assumptions the overall dependence on the temperature is in agreement with the more rigorous treatment of ${ }^{27}$. However, this expression does not involve any dependence on the different translational and rotational temperatures. Thus, in the recent literature, formulas derived from data fitting, either from numerical or experimental results, have been employed. In ${ }^{31-33}$ the following expression for the collision number is presented to be used with the Rykov model

$$
\begin{aligned}
& Z_{r}^{R y k .}=\frac{3}{4} \pi \frac{\psi(\tilde{T})}{\tilde{T}^{1 / 6}} \frac{9 \tilde{T}}{\tilde{T}+8} \frac{T_{r}}{T_{t}}\left[0.461+0.5581\left(\frac{T_{t}}{1 K}\right)+0.0358\left(\frac{T_{t}}{1 K}\right)^{2}\right] \\
& \psi(\tilde{T})=0.767+0.233 \tilde{T}^{-1 / 6} \exp (-1.17[\tilde{T}-1]) \\
& \tilde{T}=T_{t} / T^{*}
\end{aligned}
$$

An alternative expression for $Z_{r}\left(T_{t}, T_{r}\right)$ derived from molecular dynamics simulations can be found in ${ }^{42}$ as

$$
Z_{r}^{\text {Val. }}=\left[a_{1}\left(\frac{T_{t}}{1 K}\right)^{1 / 4}+a_{2}\left(\frac{T_{t}}{1 K}\right)^{-1 / 4}-a_{3}\left(\frac{T_{t}}{1 K}-1000\right)\right]\left[1-b\left(1-\frac{T_{r}}{T_{t}}\right)\right]
$$


where $a_{1}=1.33868, a_{2}=-6.19992, a_{3}=-0.00107942$ and $0<b \leq 1$. It is important to notice that, considering the moments of the Rykov model collision term, the relaxation process in the model is described as

$$
\frac{\rho\left(T-T_{r}\right)}{Z_{r} \tau}
$$

while in ${ }^{29,42}$ Jeans' equation is considered, leading to

$$
\frac{\rho\left(T_{t}-T_{r}\right)}{Z_{r} \tau}
$$

This means that the collision number in the Rykov model results

$$
Z_{r}=0.6 \times Z_{r}^{\text {Par. } / \text { Val. }} .
$$

For the viscosity law, Rykov and his co-workers ${ }^{31-33}$ suggest

$$
\mu\left(T_{t}\right)=\mu\left(T^{*}\right) \frac{\tilde{T}^{2 / 3}}{\psi(\tilde{T})}
$$

otherwise a simpler power law with an exponential factor of $0.72^{22}$ can be employed.

To make the system (7) complete, the value of the constants $\delta, \omega_{0}$ and $\omega_{1}$, need to be determined. In ${ }^{24} \omega_{0}=$ 0.2354 and $\omega_{1}=0.3049$ or $\omega_{0}=0.5$ and $\omega_{1}=0.286$ are given for diatomic gases. Both pairs of values have been successfully employed in ${ }^{23,24,32,33}$ with $\delta^{-1}=1.55$. In the present work the values $\omega_{0}=0.5$ and $\omega_{1}=0.286$ are employed.

The dimensionless macroscopic quantities can be obtained from $F_{0}$ and $F_{1}$ by means of the following formulas

$$
\begin{array}{ll}
\rho=\int_{-\infty}^{+\infty} F_{0} d \mathbf{c} & ; \rho u_{x}=\int_{-\infty}^{+\infty} c_{x} F_{0} d \mathbf{c} ; \frac{3}{2} \rho T_{t}+\rho u_{x}^{2}=\int_{-\infty}^{+\infty} c^{2} F_{0} d \mathbf{c} \\
\rho T_{r}=\int_{-\infty}^{+\infty} F_{1} d \mathbf{c} & ; \quad \frac{5}{2} T=\frac{3}{2} T_{t}+T_{r} \quad ; \quad p_{t}=\rho T_{t} \quad ; \quad p=\rho T \\
q_{x}^{t}=\int_{-\infty}^{+\infty}{c^{\prime}}^{\prime} \frac{\mathbf{c}^{\prime 2}}{2} F_{0} d \mathbf{c} \quad ; \quad q_{x}^{r}=\int_{-\infty}^{+\infty} \frac{c^{\prime} x}{2} F_{1} d \mathbf{c} .
\end{array}
$$

\subsection{The BGK-NS Method and the Unified Gas-Kinetic Scheme}

First examples of gas-kinetic schemes can be found in ${ }^{30}$ and ${ }^{28}$. In these schemes, in contrast to the commonly used Roe or AUSM fluxes, a local kinetic problem around the cell interfaces is reconstructed in order to calculate the numerical fluxes in the finite volume method. This flux can include both Euler and viscous contributions but is more expensive than Roe or AUSM fluxes with central discretisation of the viscous fluxes.

Among the gas-kinetic schemes available in the literature, ${ }^{39}$, a successful approach is represented by the BGKNS method ${ }^{45,47}$ here briefly described.

Integrating in time the BGK equation ${ }^{4}$ for a one-dimensional flow in a control volume $d x$ with a continuous particle velocity $c_{x}$ and discretised space $x_{i}$ and time $t^{n}$, with step sizes of $\Delta x$ and $\Delta t$ respectively, one obtains

$$
\left.F\right|_{i} ^{n+1}=\left.F\right|_{i} ^{n}+\frac{1}{\Delta x} \int_{t^{n}}^{t^{n+1}}\left(\left.\left[c_{x} F\right]\right|_{i-1 / 2}-\left.\left[c_{x} F\right]\right|_{i+1 / 2}\right) d t+\frac{\Delta t}{2}\left(\frac{\left.F_{M}\right|_{i} ^{n+1}-\left.F\right|_{i} ^{n+1}}{\tau^{n+1}}+\frac{\left.F_{M}\right|_{i} ^{n}-\left.F\right|_{i} ^{n}}{\tau^{n}}\right)
$$

where the trapezoidal rule has been employed for the collision term integral and $\left.\left[c_{x} F\right]\right]_{i \mp 1 / 2}$ are the fluxes of the distribution function across the cell interface. Then, taking the moments $\boldsymbol{\Psi}=\left(1, c_{x}, \mathbf{c}^{2}\right)^{T}$ of equation (17), the update of the conservative variables can be found as

$$
\mathbf{W}_{i}^{n+1}=\mathbf{W}_{i}^{n}+\frac{1}{\Delta x} \int_{t^{n}}^{t^{n+1}} \int_{-\infty}^{+\infty} \boldsymbol{\Psi}\left(\left.\left[c_{x} F\right]\right|_{i-1 / 2}-\left.\left[c_{x} F\right]\right|_{i+1 / 2}\right) d \mathbf{c} d t
$$

since the compatibility conditions for the BGK model give

$$
\int_{-\infty}^{+\infty} \boldsymbol{\Psi} \frac{F_{M}-F}{\tau} d \mathbf{c}=(0,0,0)^{T}
$$


It is known that the BGK model recovers the incorrect Prandtl number in the continuum limit and for this reason a simple correction consists in scaling the energy numerical flux, as proposed by $\mathrm{Xu}^{45}$.

This approach has been applied by $\mathrm{Xu}$ and co-workers ${ }^{48,51}$ also to predict flows with internal degrees of freedom employing the following multi-temperature BGK model

$$
\begin{aligned}
& \frac{\partial F}{\partial t}+\mathbf{c} \frac{\partial F}{\partial \mathbf{x}}=\frac{F_{*}-F}{\tau}+\frac{F_{M}(T)-F_{*}}{Z_{r} \tau} \\
& F_{M}(T)=\frac{\rho}{(\pi T)^{3 / 2}} \exp \left(-\frac{\mathbf{c}^{\prime 2}}{T}\right) \\
& F_{*}=\frac{\rho}{\left(\pi T_{r}\right)^{3 / 2} \pi T_{r}} \exp \left(-\frac{\mathbf{c}^{\prime 2}}{T_{t}}-\frac{\zeta^{2}}{T_{r}}\right)
\end{aligned}
$$

where an intermediate equilibrium state, $F_{*}$, including the rotational degrees of freedom, $\zeta$, is introduced and the assumption that the fraction of collisions exciting the rotational degrees of freedom is a given constant or a function of the flow temperatures is considered as in the Rykov model. In this case only mass, momentum and total energy are conserved during a particle collision and the moments $\Psi^{\prime}=\left(1, c_{x}, \mathbf{c}^{2}, \zeta^{2}\right)^{T}$ of the collision term are given by

$$
\int_{-\infty}^{+\infty} \boldsymbol{\Psi}^{\prime}\left(\frac{F_{*}-F}{\tau}+\frac{F_{M}(T)-F_{*}}{Z_{r} \tau}\right) d \mathbf{c}=(0,0,0, S)^{T}
$$

then, the update of the macroscopic variables is

$$
\mathbf{W}_{i}^{n+1}=\mathbf{W}_{i}^{n}+\frac{1}{\Delta x} \int_{t^{n}}^{t^{n+1}} \int_{-\infty}^{+\infty} \boldsymbol{\Psi}^{\prime}\left(\left.\left[c_{x} F\right]\right|_{i-1 / 2}-\left.\left[c_{x} F\right]\right|_{i+1 / 2}\right) d \mathbf{c} d t+\frac{\Delta t}{2}\left(\mathbf{S}_{i}^{n+1}+\mathbf{S}_{i}^{n}\right)
$$

with the source terms $S$ modelled through the Landau-Teller-Jeanes-type relaxation model ${ }^{48}$.

In ${ }^{49}$ the BGK-NS method has been improved, resulting in the Unified Gas Kinetic Scheme (UGKS), and then successfully employed with the Shakhov kinetic model ${ }^{50}$ (S-UGKS). The S-UGKS involves also the update of the non-equilibrium distribution function $F_{i}^{n+1}$ and employs a discrete integration method in the phase space with $\left.c_{x}\right|_{m}$ the $m^{\text {th }}$ discrete velocity, then, equation (18) becomes

$$
\mathbf{W}_{i}^{n+1}=\mathbf{W}_{i}^{n}+\frac{1}{\Delta x} \sum_{m} \int_{t^{n}}^{t^{n+1}} \boldsymbol{\Psi}\left(\left.\left[\left.c_{x}\right|_{m} F\right]\right|_{i-1 / 2}-\left.\left[\left.c_{x}\right|_{m} F\right]\right|_{i+1 / 2}\right) d t \Delta c_{x}
$$

where the aforementioned Prandtl correction is no longer needed, with $F$ reconstructed on the basis of the equilibrium distribution function of the Shakhov model.

Recently ${ }^{26}$ a UGKS for diatomic gas flow has been developed employing the Rykov model, (7). Also in this case, as for the modified BGK model discussed above, a source term (that needs to be determined) arises in the update of the macroscopic variables as

$$
\begin{aligned}
\mathbf{W}_{i}^{n+1}= & \mathbf{W}_{i}^{n}+\frac{1}{\Delta x} \sum_{m} \int_{t^{n}}^{t^{n+1}} \boldsymbol{\Psi}_{0}\left(\left.\left[\left.c_{x}\right|_{m} F_{0}\right]\right|_{i-1 / 2}-\left.\left[\left.c_{x}\right|_{m} F_{0}\right]\right|_{i+1 / 2}\right) d t \Delta c_{x}+ \\
& +\frac{1}{\Delta x} \sum_{m} \int_{t^{n}}^{t^{n+1}} \boldsymbol{\Psi}_{1}\left(\left.\left[\left.c_{x}\right|_{m} F_{1}\right]\right|_{i-1 / 2}-\left.\left[\left.c_{x}\right|_{m} F_{1}\right]\right|_{i+1 / 2}\right) d t \Delta c_{x}+\frac{\Delta t}{2}\left(\mathbf{S}_{i}^{n+1}+\mathbf{S}_{i}^{n}\right)
\end{aligned}
$$

where $\boldsymbol{\Psi}_{0}=\left(1, c_{x}, \mathbf{c}^{2}, 0\right)^{T}$ and $\boldsymbol{\Psi}_{1}=(0,0,1,1)^{T}$.

The method is not complete till a procedure is defined to reconstruct the time-dependent gas distribution function at the cell interfaces, $\left.F\right|_{i+1 / 2}$. For most cases $26,45,47,49,50$, the following general solution of the kinetic model equation (under the assumption of a locally constant collision time) has been employed with the BGK, Shakhov and Rykov models

$$
\begin{aligned}
F\left(x_{i+1 / 2}, t,\left.c_{x}\right|_{m}\right)= & \frac{1}{\tau} \int_{t^{n}}^{t} F_{e q}\left(x_{i+1 / 2}-\left.c_{x}\right|_{m}\left(t-t^{\prime}\right), t^{\prime},\left.c_{x}\right|_{m}\right) \exp \left(-\frac{t-t^{\prime}}{\tau}\right) d t^{\prime}+ \\
& +\exp \left(-\frac{t}{\tau}\right) F^{0}\left(x_{i+1 / 2}-\left.c_{x}\right|_{m}\left(t-t^{n}\right), t^{n},\left.\mathbf{c}\right|_{m}\right)
\end{aligned}
$$


where $F_{e q}$ is the equilibrium distribution function of the employed kinetic model and $F^{0}$ the reconstructed initial state. In the early BGK-NS approach ${ }^{45}$ an equilibrium initial state is considered, while in the later version ${ }^{47}$ and its successive UGKS a non-equilibrium initial distribution function, based on the CE expansion of the kinetic model, is used to extend the validity of the scheme towards the non-equilibrium flow conditions. In the limiting case of a well-resolved flow, equation (25) assumes a simplified form; as example for the BGK model, when a non-equilibrium initial distribution is considered, it becomes

$$
F=F_{M}-\tau\left(\frac{\partial F_{M}(T)}{\partial t}+\mathbf{c} \frac{\partial F_{M}(T)}{\partial \mathbf{x}}\right)+t \frac{\partial F_{M}(T)}{\partial t} .
$$

The latter limit approach has also been applied in ${ }^{48,51}$ with the multi-temperature BGK model (20) and more recently in ${ }^{10}$ including the vibrational degrees of freedom. Recently a GKS has been proposed in ${ }^{52}$ where, in place of equations (25) or (26), an analytical solutions of the BGK equation up to third order CE expansion, which gives the Burnett equations, is used.

Considering that the assumption of a well-resolved flow rules out discontinuities in the reconstructed distribution function, equation (26) leads to simpler schemes but, strictly speaking, valid only for relatively small perturbances from equilibrium. However, it is possible to regularise the CE expansion in order to increase the validity of the approach. Indeed, as suggested in ${ }^{46}$, the collision time in equations (26) can be replaced by a generalised one which depends on not only the local macroscopic variables, but also their gradients, and is obtained in order to have the kinetic equation satisfied. In fact, if as example we substitute equation (26) with a modified collision time $\tau^{*}$ in the extended Shakhov model (3) we obtain

$$
\tau^{*}=\frac{\tau}{1+\tau\left(D^{2} F_{M}(T) M / D F_{M}(T)\right)}
$$

where in one dimension

$$
D=\frac{\partial}{\partial t}+\mathbf{c} \frac{\partial}{\partial \mathbf{x}} \quad ; \quad D^{2}=\frac{\partial^{2}}{\partial t^{2}}+2 \mathbf{c} \frac{\partial^{2}}{\partial \mathbf{x} \partial t}+\mathbf{c}^{2} \frac{\partial^{2}}{\partial \mathbf{x}^{2}} .
$$

Finally, the equilibrium distribution function, if equation (25) is employed, (or its derivatives if the limit equation (26) is used) are expressed as Taylor expansions with the coefficients that can be obtained by means of the macroscopic variables relations, as example equations (5) for the Shakhov model, and the compatibility conditions relative to the model employed, for example equations (19) for the Shakhov model.

Summarising, the BGK-NS and UGKS approaches involve the following steps:

1. reconstruction of the initial condition at the cells interface

2. evaluation of the coefficient of the Taylor expansions of the equilibrium function or its derivatives

3. calculation of the numerical fluxes of the macroscopic variables $\mathbf{W}_{i}^{n+1}$

(a) for the UGKS, calculation of the numerical fluxes of the non-equilibrium distribution function $F_{i}^{n+1}$

4. update the macroscopic variables $\mathbf{W}_{i}^{n+1}$ employing the relative equation

(a) for the UGKS, update the non-equilibrium distribution function $F_{i}^{n+1}$ employing the relative equation.

\subsection{Gas-Kinetic Scheme for Near-Continuum Flows based on the Shakhov Model}

Integrating in time the non-dimensional Shakhov model system (3), in a one-dimensional control volume $d x$ with discretised space and time steps of $\Delta x$ and $\Delta t$ respectively, and taking the moments $\Psi=\left(1, c_{x}, \mathbf{c}^{2}\right)^{T}$ of $\left.F\right|_{i} ^{n+1}$ the updated non-dimensional macroscopic variables can be obtained as in equation (23). Here we reconstruct the time dependent distribution function at the cell-faces, i.e. $\left.F\right|_{i \pm 1 / 2}(25)$, where the initial condition is given by the CE solution of the Shakhov model

$$
F=F_{S}-\tau\left[\frac{\partial F_{M}(T)}{\partial t}+\mathbf{c} \frac{\partial F_{M}(T)}{\partial \mathbf{x}}\right] .
$$

Then, for a well-resolved flow we obtain

$$
F=F^{S}-\tau\left(\frac{\partial F_{M}(T)}{\partial t}+c_{x} \frac{\partial F_{M}(T)}{\partial x}\right)+t \frac{\partial F_{M}(T)}{\partial t} .
$$


Instead of employing Taylor expansions, we proceed analytically and obtain the derivatives of the Maxwellian distribution function in terms of the macroscopic variables and their derivatives as follow

$$
\frac{\partial F_{M}}{\partial \alpha}=F_{M}\left[\frac{1}{\rho} \frac{\partial \rho}{\partial \alpha}+\frac{1}{T}\left(\frac{\mathbf{c}^{\prime 2}}{T}-\frac{3}{2}\right) \frac{\partial T}{\partial \alpha}+2 \frac{c^{\prime}{ }_{x}}{T} \frac{\partial u_{x}}{\partial \alpha}\right] .
$$

These can then be evaluated at the cells interface knowing the values at the cells centres. The time and space derivative of the macroscopic variables can be linked by means of the compatibility condition for the currently employed CE expansion

$$
\int_{-\infty}^{+\infty}\left[\Psi\left(\frac{\partial F_{M}(T)}{\partial t}+c_{x} \frac{\partial F_{M}(T)}{\partial x}\right)\right] d \mathbf{c}=0
$$

Thus, we obtain

$$
\int_{-\infty}^{+\infty} \boldsymbol{\Psi} \frac{\partial F_{M}(T)}{\partial t} d \mathbf{c}=-\int_{-\infty}^{+\infty} \boldsymbol{\Psi} c_{x} \frac{\partial F_{M}(T)}{\partial x} d \mathbf{c}
$$

which results in

$$
\frac{\partial \mathbf{W}_{S}}{\partial t}=-\frac{\partial \mathbf{Q}_{S}}{\partial x}
$$

with

$$
\mathbf{W}_{S}=\left(\rho, \rho u_{x}, \frac{3}{2} \rho T u_{x}+\rho u_{x}^{2}\right)^{T} \quad ; \quad \mathbf{Q}_{s}=\left(\rho u_{x}, \frac{1}{2} \rho T+\rho u_{x}^{2}, \frac{5}{2} \rho T u_{x}+\rho u_{x}^{3}\right)^{T} .
$$

as discussed in ${ }^{15}$.

Introducing its CE solution (29) with a modified collision time $\tau^{*}$

$$
F=F_{S}-\tau^{*}\left(\frac{\partial F_{M}(T)}{\partial t}+\mathbf{c} \frac{\partial F_{M}(T)}{\partial \mathbf{x}}\right)
$$

in the Shakhov model (3), the following relation can be obtained

$$
\tau^{*}=\frac{\tau D F_{S}}{D F_{M}(T)+\tau D^{2} F_{M}(T)} .
$$

Assuming that the particle collision times are independent of the particle velocity, we can take moments of equation (37). We decide to take the moment $c^{\prime}{ }_{x} c^{\prime 2}$, thus

$$
\tau^{*}=\frac{\tau\left\langle D F_{S}\right\rangle}{\left\langle D F_{M}(T)\right\rangle+\tau\left\langle D^{2} F_{M}(T)\right\rangle}
$$

Since the latter $\tau^{*}$ represents a numerical evaluation of the CE expansion closure we decide to simplify the approach here neglecting the terms relative to the Prandtl number correction, thus

$$
\tau^{*}=\frac{\tau}{1+\tau \frac{\left\langle D^{2} F_{M}(T)\right\rangle}{\left\langle D F_{M}(T)\right\rangle}}
$$

where

$$
\begin{aligned}
&\left\langle D F_{M}(T)\right\rangle= \frac{5}{4} \frac{\partial\left(\rho T^{2}\right)}{\partial x}+\frac{5}{2} \rho T\left(\frac{\partial u_{x}}{\partial t}+u_{x} \frac{\partial u_{x}}{\partial x}\right) \\
&\left\langle D^{2} F_{M}(T)\right\rangle= \frac{5}{2}\left(\frac{\partial^{2}\left(\rho T^{2}\right)}{\partial t \partial x}+\frac{\partial^{2}\left(\rho T^{2} u_{x}\right)}{\partial x^{2}}\right)+ \\
&+T\left(5 \frac{\partial \rho}{\partial t} \frac{\partial u_{x}}{\partial t}+\frac{5}{2} u_{x}\left(\frac{\partial \rho}{\partial t} \frac{\partial u_{x}}{\partial x}+\frac{\partial \rho}{\partial x} \frac{\partial u_{x}}{\partial t}\right)+\left(\frac{11}{2} T+5 u_{x}^{2}\right) \frac{\partial \rho}{\partial x} \frac{\partial u_{x}}{\partial x}\right)+ \\
&+\rho T\left(5 \frac{\partial T}{\partial t} \frac{\partial u_{x}}{\partial t}+\frac{5}{2} u_{x}\left(\frac{\partial T}{\partial t} \frac{\partial u_{x}}{\partial x}+\frac{\partial T}{\partial x} \frac{\partial u_{x}}{\partial t}\right)+\left(11 T+5 u_{x}^{2}\right) \frac{\partial T}{\partial x} \frac{\partial u_{x}}{\partial x}\right)+ \\
&+\rho T\left(\frac{5}{2}\left(\frac{\partial u_{x}}{\partial t}\right)^{2}+\frac{5}{2} u_{x} \frac{\partial^{2} u_{x}}{\partial t \partial x}+8 \frac{\partial u_{x}}{\partial t} \frac{\partial u_{x}}{\partial x}+\left(\frac{11}{4} T+\frac{5}{2} u_{x}^{2}\right) \frac{\partial^{2} u_{x}}{\partial^{2} x}+16 u_{x}\left(\frac{\partial u_{x}}{\partial x}\right)^{2}\right) .
\end{aligned}
$$


The required second derivatives $\partial^{2} / \partial^{2} t$ and $\partial^{2} / \partial t \partial x$ can be expressed in terms of spatial derivatives thanks to the compatibility conditions (34)

$$
\begin{aligned}
& \frac{\partial^{2} \mathbf{W}_{S}}{\partial t \partial x}=-\frac{\partial^{2} \mathbf{Q}_{S}}{\partial x^{2}} \\
& \frac{\partial^{2} \mathbf{W}_{S}}{\partial^{2} t}=-2 \int_{-\infty}^{+\infty} \boldsymbol{\Psi} c_{x} \frac{\partial^{2} F_{M}(T)}{\partial t \partial x} d \mathbf{c}-\int_{-\infty}^{+\infty} \boldsymbol{\Psi} c_{x}^{2} \frac{\partial^{2} F_{M}(T)}{\partial^{2} x} d \mathbf{c}=-\frac{\partial^{2} \mathbf{Q}_{S}^{\prime}}{\partial t \partial x}-\frac{\partial^{2} \mathbf{Q}^{\prime \prime} S}{\partial x^{2}}
\end{aligned}
$$

where $W_{S}$ and $Q_{S}$ are defined in equations (35) and

$$
\mathbf{Q}_{S}^{\prime}=\left(2 \rho u_{x}, \rho T+2 \rho u_{x}^{2}\right)^{T} \quad ; \quad \mathbf{Q}^{\prime \prime}{ }_{S}=\left(\frac{1}{2} \rho T+\rho u_{x}^{2}, \frac{2}{2} \rho T u_{x}+\rho u_{x}^{3}\right)^{T} .
$$

Finally, similar to ${ }^{48,51}$, since $\left\langle D F_{M}(T)\right\rangle$ and $\left\langle D^{2} F_{M}(T)\right\rangle$ will be sensitive to numerical errors (especially close to equilibrium regions where they tend to vanish) a limiter is needed. In the current work, the following non-linear limiter is used

$$
\tau^{*}=\frac{\tau}{1+\max \left(-0.5, \min \left(-0.5(1.0-\exp (-c \times K n)), \tau \frac{\left\langle D^{2} F_{M}(T)\right\rangle}{\left\langle D F_{M}(T)\right\rangle}\right)\right)} .
$$

where $K n$ is the local Knudsen number based on the gradients of the macroscopic variables 9

$$
K n=\lambda \times \max \left(\frac{|\Delta \rho|}{\rho}, \frac{\left|\Delta u_{x}\right|}{u_{x}}, \frac{|\Delta T|}{T}\right)
$$

and the function $f(K n)=-0.5(1.0+\exp (-c \times K n))$ is used to obtain a smoother transition from $\tau^{*}=\tau$ to $\tau^{*}=2 \times \tau$. Here, the parameter $c=4.01341$ is calculated in order to have at least a starting $\tau^{*}=1.1 \times \tau$ at $K n=0.05$.

\subsection{Gas-Kinetic Scheme for Near-Continuum Flows based on the Rykov Model}

Integrating in time the non-dimensional reduced Rykov model system (7) and taking the moments $\boldsymbol{\Psi}_{0}=\left(1, c_{x}, \mathbf{c}^{2}, 0\right)^{T}$ of $F_{0} l_{i}^{n+1}$ and $\boldsymbol{\Psi}_{1}=(0,0,1,1)^{T}$ of $\left.F_{1}\right|_{i} ^{n+1}$, equation (24) with the following source term

$$
\frac{\Delta t}{2}\left(\mathbf{S}_{i}^{n+1}+\mathbf{S}_{i}^{n}\right)=\frac{\Delta t}{2}\left(0,0,0, \frac{\rho\left(\left.T\right|_{i} ^{n+1}-\left.T_{r}\right|_{i} ^{n+1}\right)}{Z_{r} \tau_{i}^{n+1}}+\frac{\rho\left(\left.T\right|_{i} ^{n}-\left.T_{r}\right|_{i} ^{n}\right)}{Z_{r} \tau_{i}^{n}}\right)^{T}
$$

can be obtained for the update of the non-dimensional macroscopic variables. As previously shown in ${ }^{15}$, reconstructing the time dependant distribution functions at the cell-faces, i.e. $\left.F_{0}\right|_{i \pm 1 / 2}$ and $\left.F_{1}\right|_{i \pm 1 / 2}$, consistently on the basis of the CE solution of the Rykov model

$$
\begin{aligned}
& F_{0}=F_{0}^{e q}-\tau\left[\frac{\partial F_{M}\left(T_{t}\right)}{\partial t}+\mathbf{c} \frac{\partial F_{M}\left(T_{t}\right)}{\partial \mathbf{x}}\right] \\
& F_{1}=F_{1}^{e q}-\tau\left[\frac{\partial\left(T_{r} F_{M}\left(T_{t}\right)\right)}{\partial t}+\mathbf{c} \frac{\partial\left(T_{r} F_{M}\left(T_{t}\right)\right)}{\partial \mathbf{x}}\right] .
\end{aligned}
$$

for a well-resolved flow it is possible to obtain

$$
\begin{aligned}
& F_{0}=F_{0}^{e q}-\tau\left(\frac{\partial F_{M}\left(T_{t}\right)}{\partial t}+c_{x} \frac{\partial F_{M}\left(T_{t}\right)}{\partial x}\right)+t \frac{\partial F_{M}\left(T_{t}\right)}{\partial t} \\
& F_{1}=F_{1}^{e q}-\tau\left(\frac{\partial\left(T_{r} F_{M}\left(T_{t}\right)\right)}{\partial t}+c_{x} \frac{\partial\left(T_{r} F_{M}\left(T_{t}\right)\right)}{\partial x}\right)+t \frac{\partial\left(T_{r} F_{M}\left(T_{t}\right)\right)}{\partial t}
\end{aligned}
$$

where the derivatives are obtained analytically with the derivative of the Maxwellian defined in equation (31) and

$$
F_{0}^{e q}=F_{0}^{t}+\frac{F_{0}^{r}-F_{0}^{t}}{Z_{r}} \quad ; \quad F_{1}^{e q}=F_{1}^{t}+\frac{F_{1}^{r}-F_{1}^{t}}{Z_{r}} .
$$

The time derivatives of the macroscopic variables can be obtained in terms of the space derivatives by means of the compatibility condition for the Rykov model

$$
\int_{-\infty}^{+\infty}\left[\boldsymbol{\Psi}_{0}\left(\frac{\partial F_{M}\left(T_{t}\right)}{\partial t}+c_{x} \frac{\partial F_{M}\left(T_{t}\right)}{\partial x}\right)+\Psi_{1}\left(\frac{\partial T_{r} F_{M}\left(T_{t}\right)}{\partial t}+c_{x} \frac{\partial T_{r} F_{M}\left(T_{t}\right)}{\partial x}\right)\right] d \mathbf{c}=\mathbf{S}
$$


then,

$$
\int_{-\infty}^{+\infty}\left[\boldsymbol{\Psi}_{0} \frac{\partial F_{M}\left(T_{t}\right)}{\partial t}+\boldsymbol{\Psi}_{1} \frac{\partial T_{r} F_{M}\left(T_{t}\right)}{\partial t}\right] d \mathbf{c}=\mathbf{S}-\int_{-\infty}^{+\infty}\left(\boldsymbol{\Psi}_{0} c_{x} \frac{\partial F_{M}\left(T_{t}\right)}{\partial x}+\boldsymbol{\Psi}_{1} c_{x} \frac{\partial T_{r} F_{M}\left(T_{t}\right)}{\partial x}\right) d \mathbf{c}
$$

which, as explained in ${ }^{15}$, leads to

$$
\frac{\partial \mathbf{W}_{\mathbf{R}}}{\partial t}=\mathbf{S}-\frac{\partial \mathbf{Q}_{\mathbf{R}}}{\partial x}
$$

where

$$
\mathbf{W}_{\mathbf{R}}=\left(\rho, \rho u_{x}, \frac{5}{2} \rho T+\rho u_{x}^{2}, \rho T_{r}\right)^{T} \quad ; \quad \mathbf{Q}_{\mathbf{R}}=\left[\begin{array}{c}
\rho u_{x} \\
\frac{1}{2} \rho T_{t}+\rho u_{x}^{2} \\
\frac{5}{2} \rho T_{t} u_{x}+\rho u_{x}^{3}+\rho T_{r} u_{x} \\
\rho T_{r} u_{x}
\end{array}\right] .
$$

As for the monoatomic case, if we introduce the CE solutions (46) of the Rykov model (7) with a modified collision time $\tau^{*}$

$$
\begin{aligned}
& F_{0}=F_{0}^{e q}-\tau^{*}\left(\frac{\partial F_{M}\left(T_{t}\right)}{\partial t}+\mathbf{c} \frac{\partial F_{M}\left(T_{t}\right)}{\partial \mathbf{x}}\right) \\
& F_{1}=F_{1}^{e q}-\tau^{*}\left(\frac{\partial\left(T_{r} F_{M}\left(T_{t}\right)\right)}{\partial t}+c_{x} \frac{\partial\left(T_{r} F_{M}\left(T_{t}\right)\right)}{\partial x}\right)
\end{aligned}
$$

back in the model equation, it is possible to obtain

$$
\tau^{*} D F_{M}\left(T_{t}\right)+\tau^{*} \tau D^{2} F_{M}\left(T_{t}\right)=\tau D F_{0}^{e q} \quad ; \quad \tau^{*} D\left(T_{r} F_{M}\left(T_{t}\right)\right)+\tau^{*} \tau D^{2}\left(T_{r} F_{M}\left(T_{t}\right)\right)=\tau D F_{1}^{e q} .
$$

Since the difference in the relaxation rate between translational and rotational processes is inherited in the collision number, $Z_{r}$, we define a single modified collision time by taking the moment relative to the total heat flux. Thus, multiplying the first of equations (54) by $c^{\prime}{ }_{x} c^{\prime 2}$ and the second one by $c^{\prime}{ }_{x}$, and adding the two resulting equations we obtain

$$
\tau^{*}=\frac{\tau\left(\left\langle D F_{0}^{e q}\right\rangle+\left\langle D F_{1}^{e q}\right\rangle\right)}{\left(\left\langle D F_{M}\left(T_{t}\right)\right\rangle+\left\langle D\left\{T_{r} F_{M}\left(T_{t}\right)\right\}\right\rangle\right)+\tau\left(\left\langle D^{2} F_{M}\left(T_{t}\right)\right\rangle+\left\langle D^{2}\left\{T_{r} F_{M}\left(T_{t}\right)\right\}\right\rangle\right)} .
$$

As for the S-GKS, we simplify the numerical evaluation of this closure of the CE expansion by neglecting the terms relative to the Prandtl number correction introduced in the Rykov model, then

$$
\tau^{*}=\frac{\tau}{1+\tau \frac{\left(\left\langle D^{2} F_{M}\left(T_{t}\right)\right\rangle+\left\langle D^{2}\left\{T_{r} F_{M}\left(T_{t}\right)\right\}\right\rangle\right)}{\left(\left\langle D F_{M}\left(T_{t}\right)\right\rangle+\left\langle D\left\{T_{r} F_{M}\left(T_{t}\right)\right\}\right\rangle\right)}}
$$

where

$$
\begin{aligned}
&\left\langle D F_{M}\left(T_{t}\right)\right\rangle= \frac{5}{4} \frac{\partial\left(\rho T_{t}^{2}\right)}{\partial x}+\frac{5}{2} \rho T_{t}\left(\frac{\partial u_{x}}{\partial t}+u_{x} \frac{\partial u_{x}}{\partial x}\right) \\
&\left\langle D^{2} F_{M}\left(T_{t}\right)\right\rangle= \frac{5}{2}\left(\frac{\partial^{2}\left(\rho T_{t}^{2}\right)}{\partial t \partial x}+\frac{\partial^{2}\left(\rho T_{t}^{2} u_{x}\right)}{\partial x^{2}}\right)+ \\
&+T_{t}\left(5 \frac{\partial \rho}{\partial t} \frac{\partial u_{x}}{\partial t}+\frac{5}{2} u_{x}\left(\frac{\partial \rho}{\partial t} \frac{\partial u_{x}}{\partial x}+\frac{\partial \rho}{\partial x} \frac{\partial u_{x}}{\partial t}\right)+\left(\frac{11}{2} T_{t}+5 u_{x}^{2}\right) \frac{\partial \rho}{\partial x} \frac{\partial u_{x}}{\partial x}\right)+ \\
&+\rho\left(5 \frac{\partial T_{t}}{\partial t} \frac{\partial u_{x}}{\partial t}+\frac{5}{2} u_{x}\left(\frac{\partial T_{t}}{\partial t} \frac{\partial u_{x}}{\partial x}+\frac{\partial T_{t}}{\partial x} \frac{\partial u_{x}}{\partial t}\right)+\left(11 T_{t}+5 u_{x}^{2}\right) \frac{\partial T_{t}}{\partial x} \frac{\partial u_{x}}{\partial x}\right)+ \\
&+\rho T_{t}\left(\frac{5}{2}\left(\frac{\partial u_{x}}{\partial t}\right)^{2}+\frac{5}{2} u_{x} \frac{\partial^{2} u_{x}}{\partial t \partial x}+8 \frac{\partial u_{x}}{\partial t} \frac{\partial u_{x}}{\partial x}+\left(\frac{11}{4} T_{t}+\frac{5}{2} u_{x}^{2}\right) \frac{\partial^{2} u_{x}}{\partial^{2} x}+16 u_{x}\left(\frac{\partial u_{x}}{\partial x}\right)^{2}\right)
\end{aligned}
$$


and

$$
\begin{aligned}
\left\langle D\left\{T_{r} F_{M}\left(T_{t}\right)\right\}\right\rangle= & \frac{1}{2} \frac{\partial\left(\rho T_{r} T_{t}\right)}{\partial x}+\rho T_{r}\left(\frac{\partial u_{x}}{\partial t}+u_{x} \frac{\partial u_{x}}{\partial x}\right) \\
\left\langle D^{2}\left\{T_{r} F_{M}\left(T_{t}\right)\right\}\right\rangle= & \frac{\partial^{2}\left(\rho T_{r} T_{t}\right)}{\partial t \partial x}+\frac{\partial^{2}\left(\rho T_{r} T_{t} u_{x}\right)}{\partial x^{2}}+ \\
& +T_{r}\left(2 \frac{\partial \rho}{\partial t} \frac{\partial u_{x}}{\partial t}+2 u_{x} \frac{\partial \rho}{\partial t} \frac{\partial u_{x}}{\partial x}+2 u_{x} \frac{\partial \rho}{\partial x} \frac{\partial u_{x}}{\partial t}+\left(T_{t}+2 u_{x}^{2}\right) \frac{\partial \rho}{\partial x} \frac{\partial u_{x}}{\partial x}\right)+ \\
& +\rho T_{r} \frac{\partial T_{t}}{\partial x} \frac{\partial T_{r}}{\partial x}+ \\
& +\rho\left(2 \frac{\partial T_{r}}{\partial t} \frac{\partial u_{x}}{\partial t}+2 u_{x} \frac{\partial T_{r}}{\partial t} \frac{\partial u_{x}}{\partial x}+2 u_{x} \frac{\partial T_{r}}{\partial x} \frac{\partial u_{x}}{\partial t}+\left(T_{t}+2 u_{x}^{2}\right) \frac{\partial \rho}{\partial x} \frac{\partial u_{x}}{\partial x}\right)^{2}+ \\
& +\rho T_{r}\left(\frac{\partial^{2} u_{x}}{\partial^{2} t}+2 u_{x} \frac{\partial^{2} u_{x}}{\partial t \partial x}+4 \frac{\partial u_{x}}{\partial t} \frac{\partial u_{x}}{\partial x}+\left(\frac{T_{t}}{2}+u_{x}^{2}\right) \frac{\partial^{2} u_{x}}{\partial^{2} x}+4 u_{x}\left(\frac{\partial u_{x}}{\partial x}\right)^{2}\right) .
\end{aligned}
$$

The second derivatives $\partial^{2} / \partial t \partial x$ can be expressed in terms of only spatial derivatives thanks to the compatibility conditions (50)

$$
\frac{\partial^{2} \mathbf{W}_{R}}{\partial t \partial x}=\frac{\partial \mathbf{S}}{\partial x}-\frac{\partial^{2} \mathbf{Q}_{R}}{\partial x^{2}}
$$

where $W_{R}$ and $Q_{R}$ are defined in equations (52), while the second time derivative of the mean velocity can be obtained considering the second order compatibility conditions for the translational part, equations (41) and (42) with $T=T_{t}$. Finally, the same limiter employed for the monoatomic case in equation (43) is used here with the local Knudsen number defined as in 20,34

$$
K n=\lambda \times \max \left(\frac{|\Delta \rho|}{\rho}, \frac{\left|\Delta u_{x}\right|}{u_{x}}, \frac{\left|\Delta T_{t}\right|}{T_{t}}, \frac{\left|\Delta T_{r}\right|}{T_{r}}, \frac{\left|T_{t}-T_{r}\right|}{2 T_{r}}\right)
$$

\subsection{Diffuse Wall Boundary Conditions}

The gas evolution at a solid boundary is modelled assuming that particles hit the wall with a distribution function according to the flow conditions whereas they are reflected with:

- a Maxwellian distribution according to the wall state for fully accommodation boundary (viscous wall),

- the same distribution function for specular reflection boundary (inviscid wall),

- a combination of diffuse and specular boundaries depending on the accommodation coefficient $\sigma$.

Therefore, the final gas distribution function at the wall can be written as

$$
F=\sigma F_{M u>0}+F_{u<0}+(1-\sigma) F_{u>0}
$$

where $u<0$ and $u>0$ represent the velocities of particles hitting the wall and reflected by the wall respectively; while $F_{M}$ and $F$ are the Maxwellian and the non-equilibrium distribution functions at the wall. The fluid state at the wall can be extrapolated from the domain. In the present work fully accommodated walls have been employed, i.e. $\sigma=1$.

\section{Results and Discussion}

\subsection{Evaluation of the S/R-GKS}

To evaluate the GKS, preliminary tests have been conducted on normal shocks at different Mach numbers, for both monoatomic (Argon) and diatomic (Nitrogen) gases. Details about the grid and the velocity space used can be found in table 1 . The results have been compared with the experimental data and the DSMC results presented in ${ }^{1}$. As shown in figures 2a, 3a and 4a the GKS is able to resolve shock structures with and without rotational non-equilibrium. 


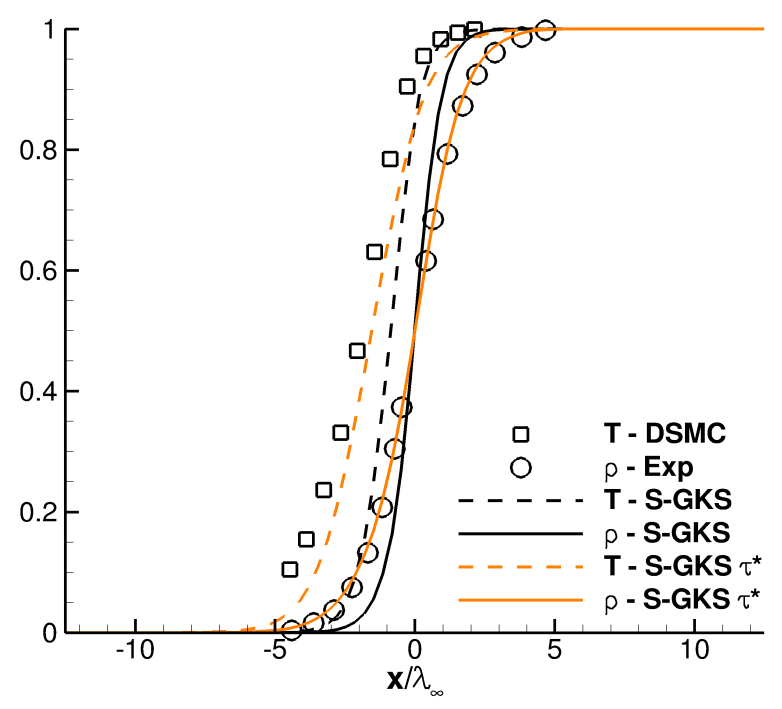

(a) $\mathrm{Mach}=3.8$.

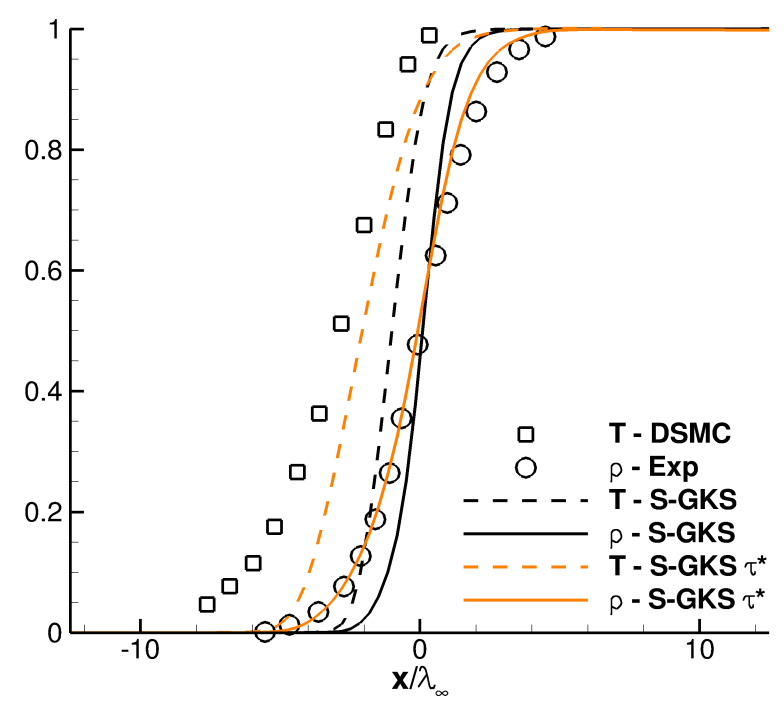

(b) $\mathrm{Mach}=9$.

Figure 2 - Non-dimensional $\rho$ and $T$ normalised profiles, i.e. $\left(\phi-\phi_{-\infty}\right) /\left(\phi_{\infty}-\phi_{-\infty}\right)$, for normal shocks in a monoatomic gas. Experimental results from ${ }^{1}$.

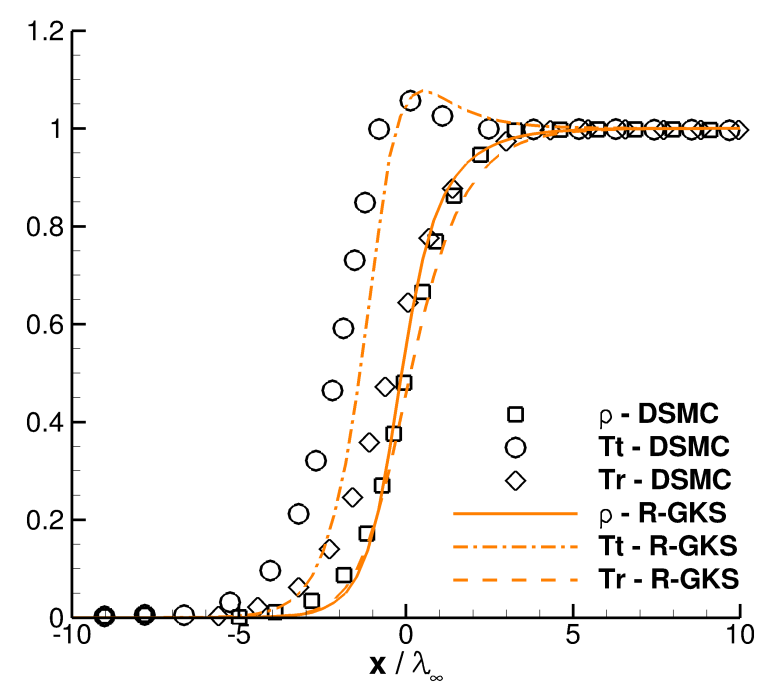

(a) Mach $=2.8$, constant $Z_{r}=4.2$, viscosity power law with exponential 0.72 .

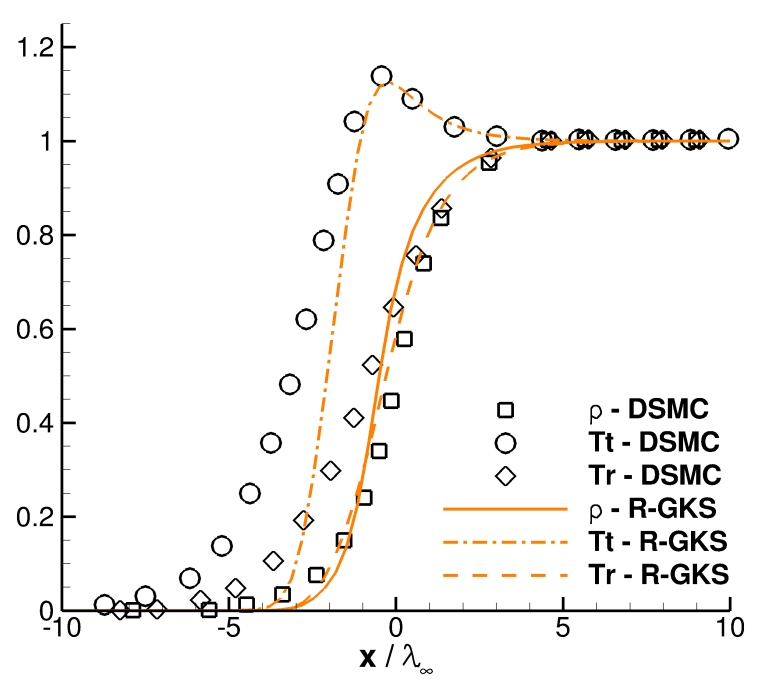

(b) Mach $=10$, constant $Z_{r}=5$, viscosity power law with exponential 0.72 .

Figure 3 - Non-dimensional $\rho, T_{t}$ and $T_{r}$ normalised profiles, i.e. $\left(\phi-\phi_{-\infty}\right) /\left(\phi_{\infty}-\phi_{-\infty}\right)$, for normal shocks in a diatomic gas. DSMC results from ${ }^{1}$.

However, due to the continuum formulation, it predicts steeper shocks. Shock-structure predictions in better agreement with DSMC and experimental results can be obtained when equation (38) or (56) is employed to modify the collision time, as can be seen in the density and temperatures profile presented in figures $2 \mathrm{a}$ and $4 \mathrm{a}$. The main differences between GKS and DSMC results can be observed for the higher Mach number cases in figures 2b, (3b) and (4b). This is probably due to the use of the CE expansion which does not allow to represent the typical bi-modal behaviour of the distribution function across shock waves at very high Mach numbers (Mach $>4)$.

Figures 5a and 5b show results for a Mach 4.89 Nitrogen flow around a flat plate with $\lambda_{\infty}=1.2 \mathrm{~mm}, T_{\infty}=116 \mathrm{~K}$ and $T_{\text {wall }}=290 \mathrm{~K}$. Grid spacing and velocity space sizes can be found in table 1 and the results are compared with the DSMC calculation and experimental data reported in ${ }^{41}$. The wall is considered fully accommodated. The GKS results prove to be in good agreement with both DVM and DSMC calculations, as seen in figure 5a. The accuracy and applicability of the GKS method has been compared with the DVM approach for the Rykov model by analysing the 


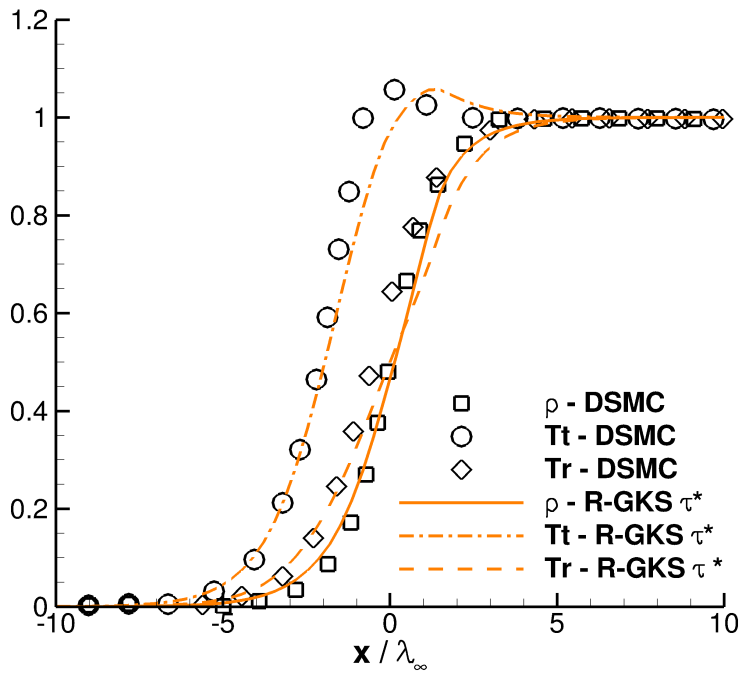

(a) Mach $=2.8$, constant $Z_{r}=4.2$, viscosity power law with exponential 0.72 .

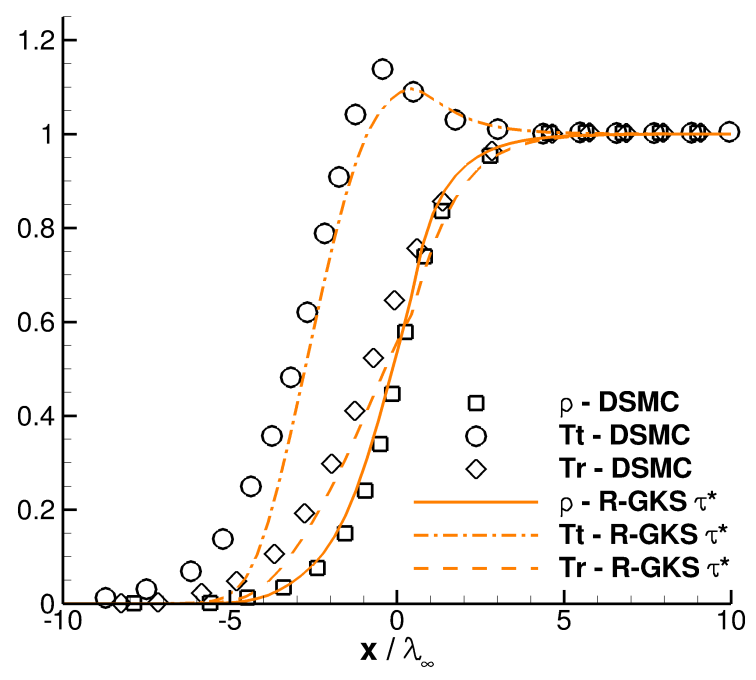

(b) Mach $=10$, constant $Z_{r}=5$, viscosity power law with exponential 0.72 .

Figure 4 - Non-dimensional $\rho, T_{t}$ and $T_{r}$ normalised profiles, i.e. $\left(\phi-\phi_{-\infty}\right) /\left(\phi_{\infty}-\phi_{-\infty}\right)$, for normal shocks in a diatomic gas. DSMC results from ${ }^{1}$.

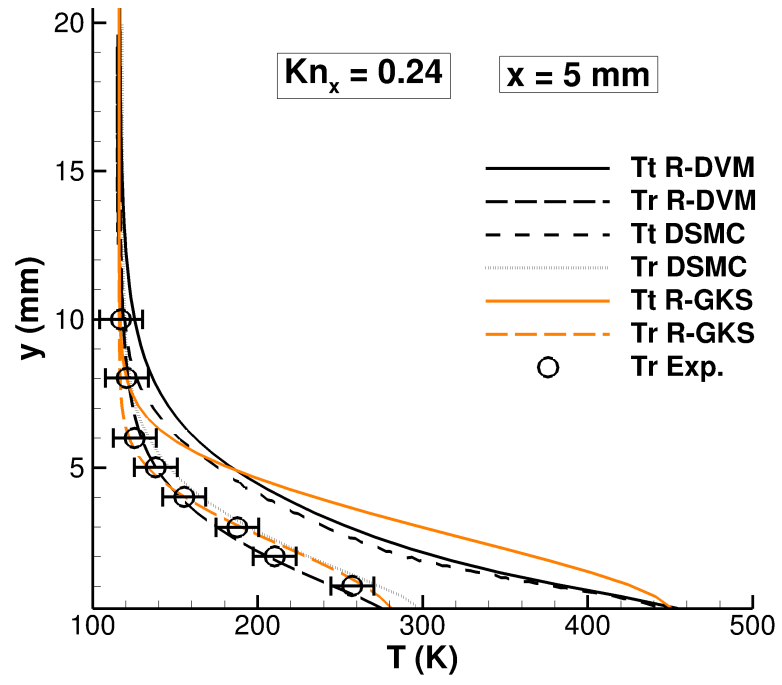

(a) $T_{t}$ and $T_{r}, K n_{x}=\lambda_{\infty} / x$. DSMC and experimental results from ${ }^{41}$

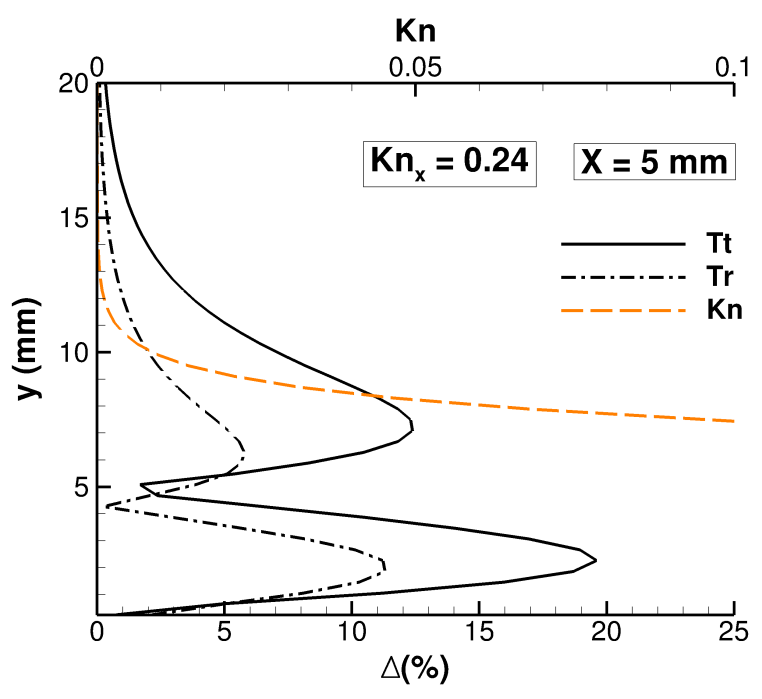

(b) R-GKS and DVM results difference and local Kn, equation (60).

Figure $5-$ Mach $=4.89$ flat plate, $Z_{r}$ equation (10) and $\mu$ equation (15).

differences between GKS and DVM results, defined here as $\Delta=\frac{\left|\phi_{D V M}-\phi_{G K S}\right|}{\left|\phi_{D V M}\right|} \times 100 \%$. A local Knudsen number, equation (60), was calculated based on the GKS solution. From this comparison for the flat plate, presented in figure $5 \mathrm{~b}$, it can be seen that the biggest difference occur when the local Knudsen number exceeds the commonly employed threshold, i.e. $0.05^{9}$. When compared with the results from Xu et al., ${ }^{48}$ as shown in figure 6, the proposed GKS is in better agreement with experimental data and DSMC results for the translational temperature at the wall. This is probably due to the fact that in the present work the GKS is based on the Rykov model which directly involves a correction that in the continuum limit leads to a variable Prandtl number, depending on the collision number $Z_{r}$, as discussed in ${ }^{15}$. Instead, in ${ }^{48}$ the scheme is based on a multi-temperature BGK model and a rescaling of the energy fluxes involving a fixed Prandtl number is employed. Therefore, considering a variable Prandtl number in region where important thermal non-equilibrium effects occur, such as the wall for the current test case, should lead to more accurate preditions. Moreover, in ${ }^{48}$ equation (9) for the collision number $Z_{r}$ is employed. The latter neglect any dependence 
on the differece between translational and rotational temperatures. As final test case a Mach 4 Nitrogen flow around a

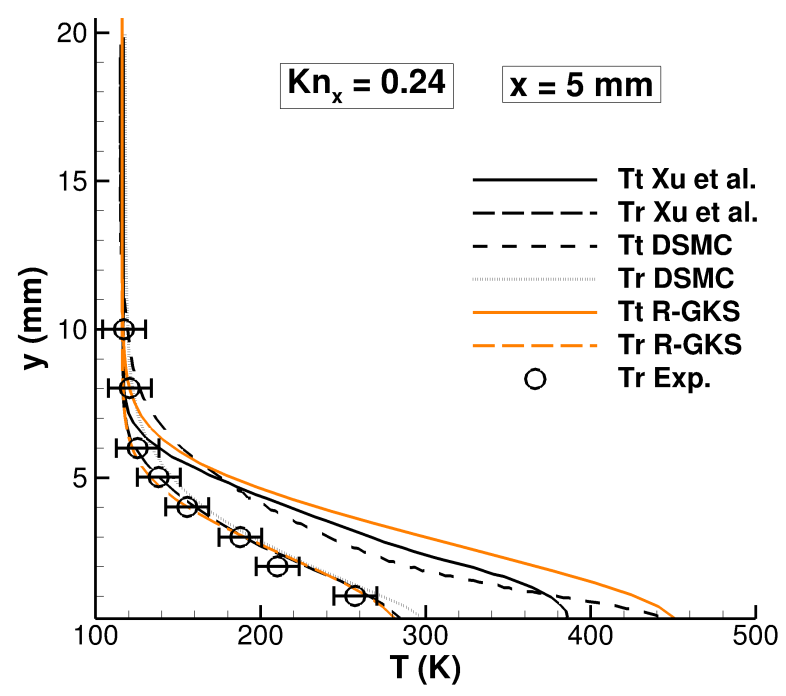

Figure $6-\mathrm{R}-\mathrm{GKS}$ and Xu et al. ${ }^{48}$ results comparison for Mach $=4.89$ flat plate, $Z_{r}$ equation (10) and $\mu$ equation (15). DSMC and experimental results from. ${ }^{41}$

$25^{\circ}$ wedge has been considered. The mesh is presented in figure $8 \mathrm{a}$; details about the calculation can be found in table 1 , while $\lambda_{\infty}=0.1 \mathrm{~mm}, T_{\infty}=185.6 \mathrm{~K}$ and $T_{\text {wall }}=293.3 \mathrm{~K}$ are the conditions. Also in this case the wall is considered fully accomodated. The DSMC, hybrid approach and continuum results reported are from ${ }^{44}$. As shown in figures $7 \mathrm{a}, 7 \mathrm{~b}$ and $8 \mathrm{~b}$ a similar behaviour is observed for the wedge case and the biggest differences between GKS and DVM results take place across the shock where the local Knudsen number is greater than 0.1 . Anyway, figures $7 \mathrm{a}$ and $7 \mathrm{~b}$

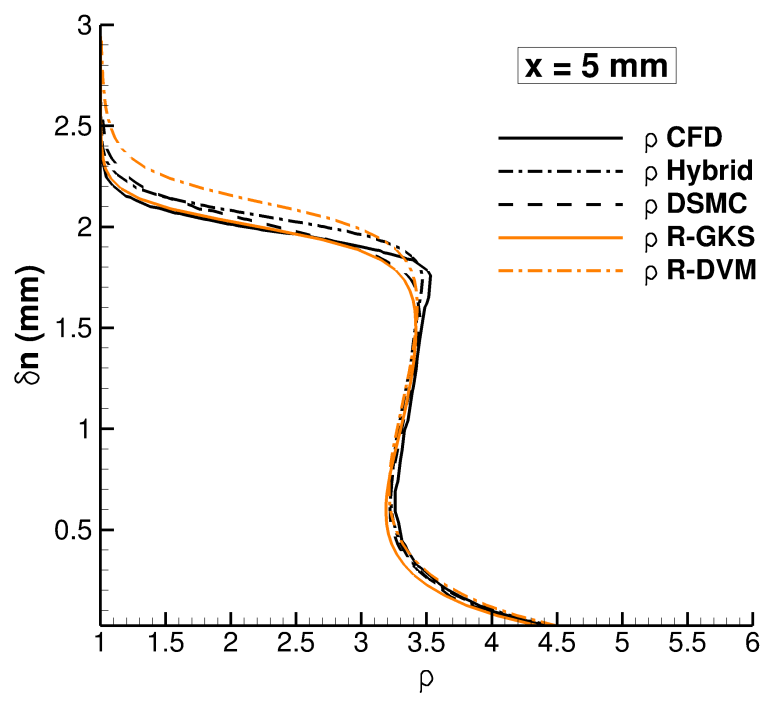

(a) Non-dimensional $\rho$.

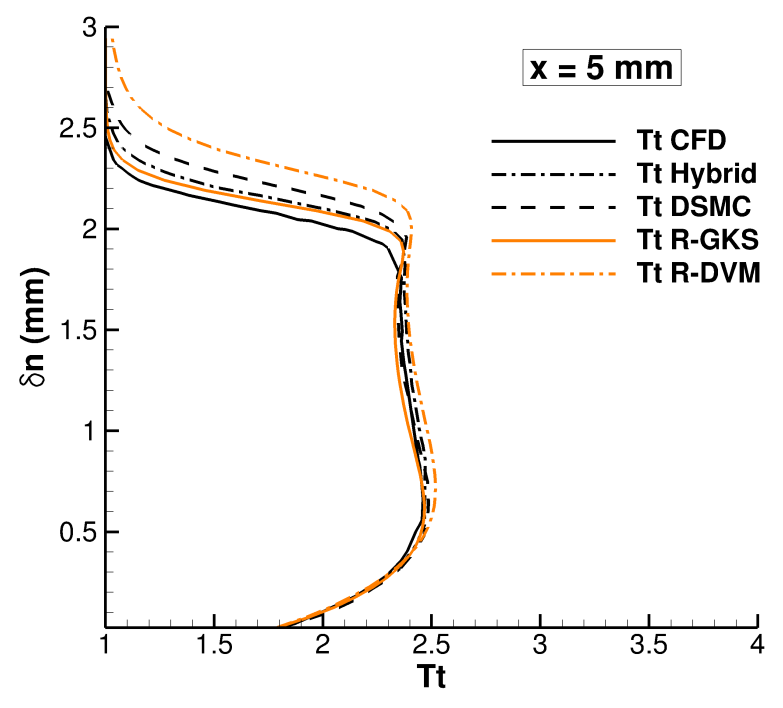

(b) Non-dimensional $T_{t}$.

Figure $7-$ Mach $=4$ wedge $25^{\circ}, Z_{r}$ equation (10) and $\mu$ equation (15). DSMC, hybrid and continuum results from. ${ }^{44}$

also show a good agreement between GKS and hybryd method results. Moreover, in both the two-dimensional cases the quantities at the wall are predicted with a difference less of 5\% relative to DVM calculations.

\subsection{Computational and Memory Cost}

The GKS is implemented so that the particle velocity space dimensions depend on the local state, while at the moment due its complexity this feature is not available in the DVM for the kinetic Boltzmann equations in $M \Phi C$. In 


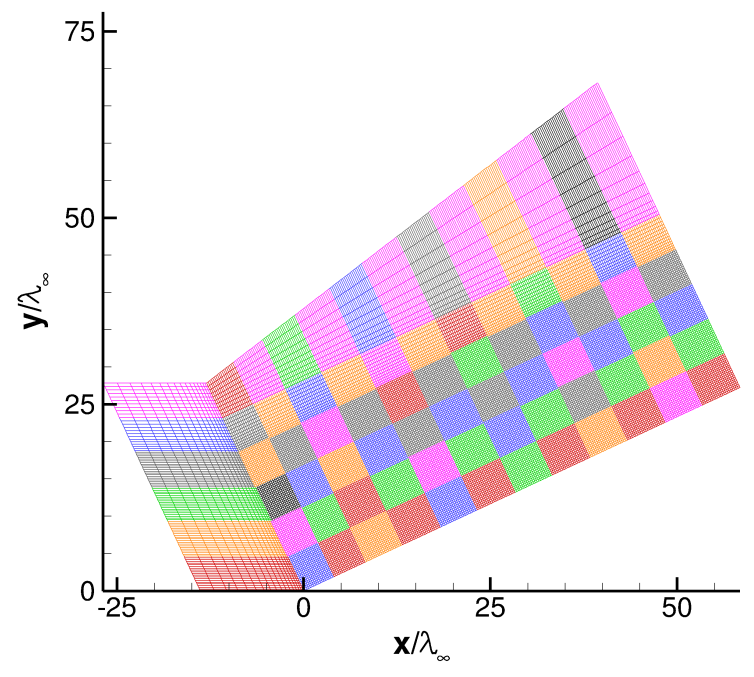

(a) Mesh.

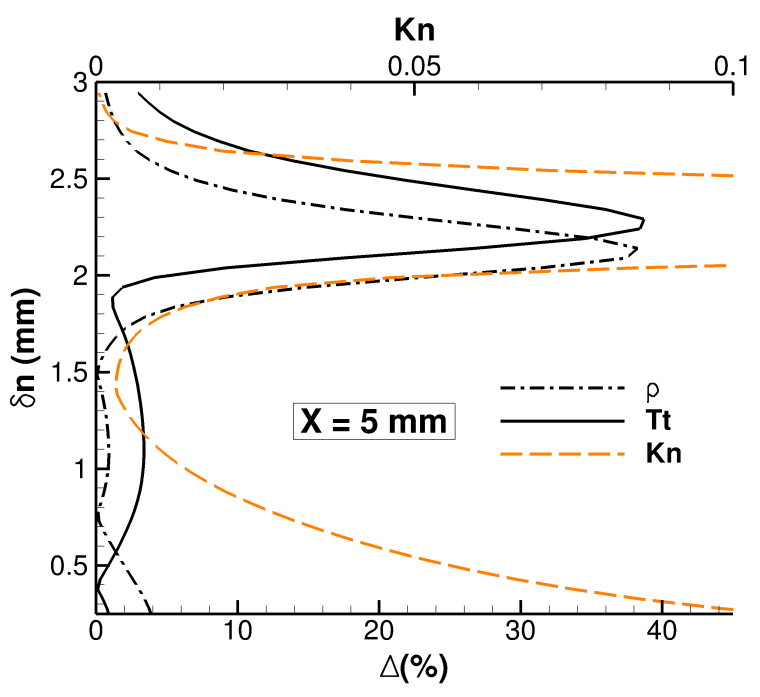

(b) R-GKS and DVM results difference and local Kn, equation (60).

Figure $8-$ Mach $=4$ wedge $25^{\circ}, Z_{r}$ equation (10) and $\mu$ equation (15).

order to perform a fair comparison about the computational time for the two approaches constant velocity spaces were used. The runs have been performed on Intel Xeon processors at the University of Liverpool cluster Chadwick and solution have been considered converged when the $L^{2}$-norm of the update between two consecutive solution is lower than $10^{-7}$ for the normal shock cases and $10^{-8}$ for the two-dimensional cases. Furthermore, it needs to be reminded that the halo exchange in the GKS involves only the flow state while the DVM needs to exchange the full velocity space and this represents an advantage of the GKS relative to the DVM and the full UGKS when a parallel calculation is performed.

\begin{tabular}{c|c|c|c|c|c|c|c|c|}
\hline \hline Test Case & Phys. Cells & $\Delta x$ & Vel. Cells & $\Delta u$ & Solver & Cores & Iterations & Time (min) \\
\hline$M=2.8$ Shock & 352 & $0.25 \lambda_{\infty}$ & $64 \times 64$ & $0.5 u_{\infty}$ & DVM & 8 & 22480 & 223 \\
\hline$M=2.8$ Shock & 352 & $0.25 \lambda_{\infty}$ & $64 \times 64$ & $0.5 u_{\infty}$ & GKS & 8 & 14016 & 10 \\
\hline$M=4.89$ Plate & 24396 & $0.25 \lambda_{\infty}$ & $32 \times 32$ & $0.5 u_{\infty}$ & DVM & 16 & 9178 & 878 \\
\hline$M=4.89$ Plate & 24396 & $0.25 \lambda_{\infty}$ & $32 \times 32$ & $0.5 u_{\infty}$ & GKS & 16 & 27555 & 363 \\
\hline$M=4.0$ Wedge & 28016 & $0.25 \lambda_{\infty}$ & $48 \times 32$ & $0.5 u_{\infty}$ & DVM & 64 & 29494 & 1925 \\
\hline$M=4.0$ Wedge & 28016 & $0.25 \lambda_{\infty}$ & $48 \times 32$ & $0.5 u_{\infty}$ & GKS & 64 & 17623 & 109 \\
\hline \hline
\end{tabular}

Table 1 - Test cases details and computational time.

In table 1 the computational time for the studied cases are reported and the GKS is found to be from $60 \%$ to $90 \%$ faster than the DVM. This is partially due to the lower number of iterations needed in general by the GKS (for the cases in table 1, 0.7 and 0.9 were the maximum CFL numbers which could be used with DVM and GKS respectively), but mainly to the time needed per iteration, around $7-10$ times smaller than the DVM. Finally, in terms of the memory cost of the GKS, the latter is drastically reduced compared with the DVM and the full UGKS. Indeed, in the DVM and the UGKS the values of the distribution function need to be stored for each physical cell in the full velocity space while the GKS being employed in the context of a continuum solver requires only the storage of the primitive variables (i.e. in contrast to the continuum solver, which typically stores 5 continuum flow quantities, the DVM and the UGKS store $\mathrm{O}\left(10^{4}\right)$ degrees of freedom per cell).

Thus, employing the GKS in place of the DVM where the fluid is near thermal equilibrium, the performance of the hybrid solver can be improved in both memory and CPU time requirements without compromising the accurancy. Moreover, extending the validity of the continuum formulation employing GKS may result in a reduction of the hybrid simulations' sensitivity to the positioning of the interfaces. 


\section{Conclusions and future works}

In the present work, an analytical GKS has been developed and added to the framework presented in ${ }^{36-38}$. The prediction of flow fields where rarefied and continuum regions coexist require the solution of two models, the NS equations and the BTE. Since the methods to solve the BTE equation are expensive, the reduction of the region where this is strictly required could improve the performance of hybrid simulations. For these reasons a GKS for nearcontinuum regime has been proposed.

The scheme has been tested for various test cases and Mach numbers proving to produce reliable predictions for near-continuum flows. Compared with a kinetic DVM solver, the near-continuum GKS solver was found to be between from $60 \%$ to $90 \%$ faster than the former. Furthermore, due to the lower number of variables that need to be stored the GKS is less expensive in terms of memory than the DVM and the full UGKS. This proves that GKS can be a viable way to improve the performance of hybrid simulations maintainig an acceptable level of reliability when used in place of more complex methods for weekly rarefied flows.

Future works will involve the evaluation of the GKS for more complex multi-dimensional flows and the implementation of the GKS in the context of hybrid simulations which will require the study of an appropriate coupling between GKS and DVM. This should allow to reduce the extent of the flow domain where the DVM is required due to the ability of the GKS to solve weakly rarefied flow conditions.

\section{References}

[1] H. Alsmeyer. Density Profiles in Argon and Nitrogen Shock Waves Measured by the Absorptiobn of an Electron Beam. Journal of Fluid Mechanics, 74(3):497-513, 1976.

[2] P. Andries, P. LeTallec, J.-P. Perlat, and B. Perthame. The Gaussian-BGK Model of Boltzmann Equation with Small Prandtl Number. European Journal of Mechanics - B/Fluids, 19(6):813-830, 2000.

[3] C. Baranger, J. Claudel, N. Hérouard, and L. Mieussens. Locally Refined Discrete Velocity Grids for Deterministic Rarefied Flow Simulations. AIP Conference Proceedings, 1501:389-396, 2012.

[4] P. Bhatnagar, E. Gross, and M. Krook. A Model for Collision Processes in Gases. I. Small Amplitude Processes in Charged and Neutral One-Component Systems. Physical Review, 94(3):511-525, 1954.

[5] G. Bird. Molecular Gas Dynamics and the Direct Simulation of Gas Flows. 1994.

[6] G. Bird. The DSMC Method. CreateSpace, 2013.

[7] J.-F. Bourgat, P. Le Tallec, and M. Tidriri. Coupling Boltzmann and Navier-Stokes Equations by Friction. Journal of Computational Physics, 127:227-245, 1996.

[8] I. Boyd. Rotational-Translational Energy Transfer in Rarefied Non-equilibrium Flows. Physics of Fluids A, 2(3), 1990.

[9] I. Boyd, G. Chen, and G. Candler. Predicting Failure of the Continuum Fluid Equations in Transitional Hypersonic Flows. Physics of Fluids, 7(1):210-219, 1995.

[10] C. Cai, D. Danny, and K. Xu. One-Dimensional Multiple-Temperature Gas-Kinetic Bhatnagar-Gross-Krook Scheme for Shock Wave Computation. AIAA Journal, 46(2):1054-1062, 2008.

[11] C. Cercignani. Theory and Application of the Boltzmann Equation. Scottish Academic Press, 1975.

[12] S. Chen, K. Xu, and Q. Cai. Expanded Analogy Between Boltzmann Kinetic Theory of Fluids and Turbulence. arXiv:1304.0865v2 [physics.flu-dyn], 2013.

[13] S. Colonia, R. Steijl, and G. Barakos. Implicit Implementation of the $\mathrm{AUSM}^{+}$and $\mathrm{AUSM}^{+}$up Schemes. International Journal for Numerical Methods in Fluids, 75(10):687-712, 2014.

[14] S. Colonia, R. Steijl, and G. Barakos. Kinetic Models with Rotational Degrees of Freedom for Hybrid Methods. In ECCOMAS - 6th European Conference on Computational Fluid Dynamics, 20-25 July, Barcelona, Spain 2014.

[15] S. Colonia, R. Steijl, and G. Barakos. Kinetic Models and Gas Kinetic Schemes for Hybrid Simulation of Partially Rarefied Flows. In AIAA Atmospheric Flight Mechanics Conference, AIAA Science and Technology Forum, 5-9 January, Kissimmee, Florida 2015. 
[16] P. Degond and G. Dimarco. Fluid Simulations with Localised Boltzmann Upscaling by Direct Simulation MonteCarlo. Journal of Computational Physics, 231:2414-2437, 2012.

[17] P. Degond, G. Dimarco, and L. Mieussens. A multiscale kinetic-fluid solver with dynamic localization of kinetic effects. Journal of Computational Physics, 229:4907-4933, 2010.

[18] P. Degond, S. Jin, and L. Mieussens. A Smooth Transition Model Between Kinetic and Hydrodnamic Equaions. Journal of Computational Phsics, 209:665-694, 2005.

[19] T. Deschenes and I. Boyd. Extension of a Modular Particle-Continuum Method to Vibrationally Exited, Hypersonic Flows. AIAA Journal, 49(9), 2011.

[20] T. Deschenes, T. Holman, and I. Boyd. Effects of Rotational Energy Relaxation in a ModularParticle-Continuum Method. Journal of Thermophysics and Heat Transfer, 25(2):218-227, 2011.

[21] C. Glass and T. Horvarth. Comparison of a 3-D CFD-DSMC Solution Methodology With a Wind Tunnel Experiment. NASA/TM-2002-211777.

[22] F. Jones. NBS TN-1186 - Interpolation Formulas for Viscosity of Six Gases: Air, Nitrogen, Carbon Dioxide,Helium, Argon, and Oxygen. U.S. Department of Commerce/National Bureau of Standards, 1984.

[23] I. Larina and V. Rykov. Computation of a Rarefied Diatomic Gas Flows through a Plane Microchannel. Computational Mathematics and Mathematical Physics, 52(4):637-648, 2010.

[24] I. Larina and V. Rykov. Kinetic Model of the Boltzmann Eqaution for a Diatomic Gas with Rotational Degrees of Freedom. Computational Mathematics and Mathematical Physics, 50(12):2118-2130, 2010.

[25] P. Le Tallec and F. Mallinger. Coupling Boltzmann and Navier-Stokes Equation by Half Fluxes. Journal of Computational Physics, 136:51-67, 1997.

[26] S. Liu, Y. Pubing, K. Xu, and C. Zhong. Unified Gas-Kinetic Scheme for Diatomic Molecular Simulations in all Flow Regimes. Journal of Computational Physics, 259:96-113, 2014.

[27] J. Lordi and R. Mates. Rotational Relaxation in Nonpolar Diatomic Gases. Physics of Fluids, 9(4):291-308, 1970.

[28] J. Mandal and S. Deshpande. Kinetic Flux Vector Splitting for Euler Equations. Computers E Fluids, 23(2):447$478,1994$.

[29] J. Parker. Rotational and Vibrational Relaxation in Diatomic Gases. Physics of Fluids, 2(4):449-462, 1959.

[30] D. Pullin. Direct Simulation Methods for Compressible Inviscid Ideal Gas Flow. Journal of Computational Physics, 34(2):231-244, 1980.

[31] V. Rykov. A Model Kinetic Equation for a Gas with Rotational Degrees of Freedom. Fluid Dynamics, 10(6):959966, 1975.

[32] V. Rykov, V. Titarev, and E. Shakhov. Numerical Study of the Transverse Supersonic Flow of a Diatomic Rarefied Gas past a Plate. Computational Mathematics and Mathematical Physics, 47(1):136-150, 2007.

[33] V. Rykov, V. Titarev, and E. Shakhov. Shock Wave Structure in a Diatomic Gas Based on a Kinetic Model. Fluid Dynamics, 43(2):316-326, 2008.

[34] T. Schwartzentruber. A Modular Particle-Continuum Numerical Algorithm for Hypersonic Non-equilibrium Flows. PhD dissertation, The Univeristy of Michigan, 2007.

[35] E. Shakhov. Generalization of the Krook Kinetic Relaxation Equation. Mekhanika Zhidkosti i Gaza, 3(5):142$145,1968$.

[36] R. Steijl and G. Barakos. Coupled Navier-Stokes-Molecular Dynamics simulations Using a Multi-physics Flow Simulation Framework. International Journal for Numerical Methods in Fluids, 62:1081-1106, 2010.

[37] R. Steijl and G. Barakos. Computational Fluid dynamics of Partially Rarefied Flows with Coupled Kinetic Boltzmann/Navier-Stokes Methods. In ECCOMAS 2012, 10-14 September, Vienna, Austria 2012. 
[38] R. Steijl and G. Barakos. Coupled Navier-Stokes/Molecular Dynamics Simulations in Nonperiodic Domains on Particle Forcing. International Journal for Numerical Methods in Fluids, 69:1326-1349, 2012.

[39] L. Tang. Progress in gas-kinetic upwind schemes for the solution of Euler/Navier-Stokes equations - I: Overview. Computers E Fluids, 56:39-48, 2012.

[40] V. Titarev, M. Dumbserc, and S. Utyuzhnikov. Construction and Comparison of Parallel Implicit Kinetic Solvers in Three Spatial Dimensions. Journal of Computational Physics, 256:17-33, 2014.

[41] N. Tsuboi and Y. Matsumoto. Experimental and Numerical Study of Hypersonic Rarefied Gas Flow over Flat Plates. AIAA Journal, 43(6):1243-1255, 2005.

[42] P. Valentini, C. Zhang, and T. Schwartzentruber. Molecular Dynamics Simulation of Rotational Relaxation in Nitrogen: Implications for Rotational Collision Number Models. Physics of Fluids, (24):106101:1-23, 2012.

[43] W. Vincenti and C. Kruger. Introduction to Physical Gas Dynamics. John Wiley and Sons, Inc., 1965.

[44] W.-L. Wang, Q. Sun, and I. Boyd. Towards Development of a Hybrid DSMC-CFD Method for Simulating Hypersonic Interacting Flows. In 8th AIAA/ASME Joint Thermophysics and Heat Transfer Conference, 24-26 June, St. Louis, Missouri 2002.

[45] K. Xu. A Gas-Kinetic BGK Scheme for the Navier-Stokes Equations and its Connection with Artificial Dissipation and Godunov Method. Journal of Computational Physics, 171:289-335, 2001.

[46] K. Xu. Regularisation of the Chapman-Enskog Expansion and its Description of Schock Structures. Physics of Fluids, 14(4):L17, 2002.

[47] K. Xu. A Multidimansional Gas-Kinetic BGK Scheme for Hypersonic Viscous Flow. Journal of Computational Physics, 203:405-421, 2005.

[48] K. Xu, X. He, and C. Cai. Multiple Temperature Kinetic Model and Gas-Kinetic Method for Hypersonic Nonequilibrium Flow Computations. Journal of Computational Physics, 227:6779-6794, 2008.

[49] K. Xu and J.-C. Huang. A Unified Gas-Kinetic Scheme for Continuum and Rarefied Flows. Journal of Computational Physics, 229:7747-7764, 2010.

[50] K. Xu and J.-C. Huang. An Improved Unified Gas-Kinetic Scheme and the Study of Shock Structures. IMA Journal of Applied Mathematics, 76:698-711, 2011.

[51] K. Xu and E. Josyula. Continuum Formulation for Non-equilibrium Shock Structure Calculation. Communications in Computational Physics, 1(3):425-450, 2006.

[52] L.-J. Xuan and K. Xu. A New Gas-Kinetic scheme Based on Qnalytical Solutions of the BGK Equation. Journal of Computational Physics, 234:524-539, 2013. 